\title{
ANALYSIS OF STEADY STATE WEAR PROCESSES FOR INHOMOGENEOUS MATERIALS AND VARYING CONTACT LOADS
}

\author{
I. PÁCZelt ${ }^{a}$, Z. Mróz ${ }^{b}$ AND A. BAKsA ${ }^{a}$ \\ ${ }^{a}$ Institute of Applied Mechanics, University of Miskolc, HUNGARY, \\ ${ }^{b}$ Institute of Fundamental Technological Research, Warsaw, POLAND \\ istvan.paczelt@uni-miskolc.hu zmroz@ippt.pan.pl attila.baksa@uni-miskolc.hu
}

[Received: December 6, 2019, Accepted: June 8, 2020]

\begin{abstract}
The transient wear process on a frictional interface of two elastic bodies in relative steady sliding motion induces shape evolution of contact interface and tends to a steady state. Then wear growth develops at constant contact stress and strain distributions. In previous papers these cases were analyzed for the fixed sliding velocity between the bodies and for fixed loads 1 . 4 . The cases of periodic sliding under fixed normal loads were treated in [5. The cases of periodic loads for fixed or varying sliding velocity were investigated in 6. The variational procedure and minimization of the response functional corresponding to the wear dissipation power were applied. The modified Archard wear rule was assumed. The specific examples were solved assuming fixed values of wear parameters in the contact domain. In the present paper the previous analyses are extended to cases when the wear parameters can vary along the sliding path and similarly, the sliding velocity and normal load can vary periodically. The cases of a ring segment-on rotating disk and translating punch-on strip are considered, providing wear analysis accounting for temperature effect.
\end{abstract}

Mathematical Subject Classification: 74M10, 74M15

Keywords: contact problems, sliding wear, steady state, variational principle, optimal contact surface, p-version of finite elements

\section{INTRODUCTION}

In the papers Páczelt and Mróz 1, 2] a new variational principle was proposed aimed at characterization of steady wear processes. It is based on the minimum principle of wear dissipation power with the equilibrium condition of interacting bodies as a constraint. In the examples treated the wear parameters were supposed to be uniform in the contact zone. In the present paper, we shall extend the analysis by considering the wear process of inhomogeneous bodies.

A class of problems will be considered when the wear parameters are not fixed but can vary with point position in the contact zone. Also elastic moduli are allowed to vary with point position in the body domain. Such assumptions provide a more realistic approach when a surface treatment is applied to improve hardness and wear resistance of contact layers. A general theoretical framework is first developed and next the numerical wear analysis is presented for two specific problems, namely a ring brake and translating punch on a plane substrate. It is demonstrated that the varying 
wear parameters affect essentially the contact pressure distribution and the wear rate in the steady state.

The wear rule specifies the wear rate $\dot{w}_{i, n}$ of the $i^{\text {th }}$ body in the normal contact direction. Following the previous work [1, 2 the modified Archard wear law is

$$
\dot{w}_{i, n}=\beta_{i}\left(\tau_{n}\right)^{b_{i}}\left\|\dot{\boldsymbol{u}}_{\tau}\right\|^{a_{i}}=\beta_{i}\left(\mu p_{n}\right)^{b_{i}}\left\|\dot{\boldsymbol{u}}_{\tau}\right\|^{a_{i}}=\beta_{i}\left(\mu p_{n}\right)^{b_{i}} v_{r}^{a_{i}}=\widetilde{\beta}_{i} p_{n}^{b_{i}} v_{r}^{a_{i}}, i=1,2
$$

where $\beta_{i}, a_{i}$ and $b_{i}$ are the wear parameters, $\mu$ is the friction coefficient, the relative tangential velocity is $\dot{\mathbf{u}}_{\tau}, \widetilde{\beta}_{i}=\beta_{i} \mu^{b_{i}}, v_{r}=\left\|\dot{\boldsymbol{u}}_{\tau}\right\|$. The shear stress at the contact surface is denoted by $\tau_{n}$ and related to the contact pressure $p_{n}$ by the Coulomb friction law $\tau_{n}=\mu p_{n}$. The unilateral contact occurs on the boundary portion $S_{c}$. In [1, 2] the wear rate vector was introduced, which is not only normal to the contact surface but also has tangential component. The elastic rates in the normal and tangential directions are denoted by $\dot{\boldsymbol{u}}_{e, n}^{(\alpha)}$ and $\dot{\boldsymbol{u}}_{e, \tau}^{(\alpha)}$, respectively. The rigid body rates are denoted by $\dot{\boldsymbol{u}}_{R, n}^{(\alpha)}, \dot{\boldsymbol{u}}_{R, \tau}^{(\alpha)}$. The relative tangential velocity has the elastic and rigid body portions

$$
\dot{\boldsymbol{u}}_{\tau}=\dot{\boldsymbol{u}}_{e, \tau}^{(2)}+\dot{\boldsymbol{u}}_{R, \tau}^{(2)}-\left(\dot{\boldsymbol{u}}_{e, \tau}^{(1)}+\dot{\boldsymbol{u}}_{R, \tau}^{(1)}\right)=\dot{\boldsymbol{u}}_{e, \tau}+\dot{\boldsymbol{u}}_{R, \tau},\left\|\dot{\boldsymbol{u}}_{\tau}\right\|=v_{r}
$$

Rigid body velocity has two components:

1. The sliding velocity is prescribed by stated boundary conditions allowing for a rigid body motion and elastic displacements developed in a stressed state.

2. The wear velocity is defined by the the rigid body motion induced by wear, so that

$$
\begin{array}{lr}
\dot{u}_{R, n}^{(w)}= & \left(\dot{\boldsymbol{\lambda}}_{F}+\dot{\boldsymbol{\lambda}}_{M} \times \Delta \boldsymbol{r}\right) \cdot \boldsymbol{n}_{c}, \\
\dot{\boldsymbol{u}}_{R, \tau}^{(w)}= & \left(\dot{\boldsymbol{\lambda}}_{F}+\dot{\boldsymbol{\lambda}}_{M} \times \Delta \boldsymbol{r}\right)-\dot{u}_{R, n} \boldsymbol{n}_{c},
\end{array}
$$

where $\dot{\boldsymbol{\lambda}}_{F}$ and $\dot{\boldsymbol{\lambda}}_{M}$ are the relative translation and rotation velocities induced by wear, which should be determined from the contact problem solution, $\Delta \mathbf{r}$ is the position vector with respect to a reference point.

Assume now that the body $B_{1}$ plays the role of an indenter and its rigid body motion is associated with the wear process. The body $B_{2}$ executes the sliding motion on the contact surface.

The contact stress of interaction of bodies $B_{1}$ and $B_{2}$ is

$$
\boldsymbol{t}^{c}=\boldsymbol{t}_{1}^{c}=-\boldsymbol{t}_{2}^{c}=-p_{n}\left(\boldsymbol{n}_{c} \pm \mu \boldsymbol{e}_{\tau 1}\right)-\mu_{d} p_{n} \boldsymbol{e}_{\tau 2}=-p_{n} \tilde{\boldsymbol{n}}_{c}
$$

where $\mu$ is the friction coefficient specifying the shear stress in sliding direction and $\mu_{d}$ is the friction coefficient associated with transverse wear velocity.

In the wear process the friction between two bodies depends not only on asperity interaction generating shear stress nearly oriented along the relative sliding velocity, but also on the direction of motion of the debris particle layer formed in the contact zone. In direction of the relative sliding velocity the friction coefficient is usually larger than in the transverse direction. In 2 three types of tangential wear dissipation models were introduced and discussed, one accounting only for wear particle slip and the other for the wear debris particle rotation and slip within the contact zone. The proper selection of the transverse friction model should be based on micromechanical 
analysis and validation tests. The other reason for non-coaxial slip and friction force action is related to the anisotropic friction condition discussed in detail in Reference [7].

The vectors $\boldsymbol{e}_{\tau 1}, \boldsymbol{e}_{\tau 2}$ and $\boldsymbol{n}_{c}$ provide the local reference triad. Here $\boldsymbol{n}_{c}$ is the unit normal to the contact surface of body $B_{1}, \boldsymbol{e}_{\tau 1}$ is the tangent unit vector coaxial with the sliding velocity and $\boldsymbol{e}_{\tau 2}$ is the transverse tangent unit vector. The sign + corresponds to the case when the relative velocity $\dot{\boldsymbol{u}}_{\tau}=\dot{\boldsymbol{u}}_{\tau}^{(2)}-\dot{\boldsymbol{u}}_{\tau}^{(1)}=-\left\|\dot{\boldsymbol{u}}_{\tau}\right\| \boldsymbol{e}_{\tau 1}=$ $-v_{r} \boldsymbol{e}_{\tau 1}$ is with the corresponding shear stress acting on the body $B_{1}$ along $-\boldsymbol{e}_{\tau 1}$.

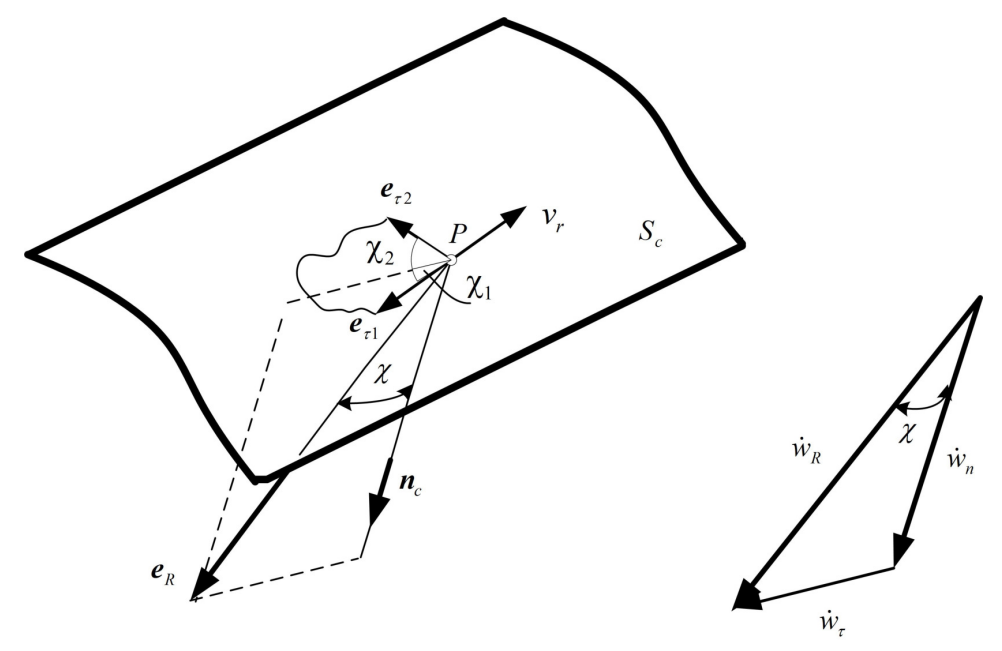

Figure 1. Wear rate vectors, local coordinate system

The wear rate vector for body $B_{i} i=1,2$ (see Figure 1 is

$$
\dot{\boldsymbol{w}}_{i}=(-1)^{i}\left(\dot{w}_{i, n} \boldsymbol{n}_{c}-\dot{w}_{i, \tau 1} \boldsymbol{e}_{\tau 1}-\dot{w}_{i, \tau 2} \boldsymbol{e}_{\tau 2}\right),
$$

where the tangential wear components can be regarded as the initial velocities for the wear debris transport in the contact layer.

A fundamental assumption was introduced, namely, at the steady state the wear rate vector is collinear with the rigid body wear velocity of $B_{1}$, so that

$$
e_{R}=\frac{\dot{\lambda}_{F}+\dot{\lambda}_{M} \times \Delta \boldsymbol{r}}{\left\|\dot{\boldsymbol{\lambda}}_{F}+\dot{\boldsymbol{\lambda}}_{M} \times \Delta \boldsymbol{r}\right\|}
$$

and

$$
\begin{gathered}
\dot{\boldsymbol{w}}=\dot{\boldsymbol{w}}_{2}-\dot{\boldsymbol{w}}_{1}=\dot{\boldsymbol{w}}_{R}=\dot{\boldsymbol{w}}_{2, R}-\dot{\boldsymbol{w}}_{1, R}=\dot{w}_{R} \boldsymbol{e}_{R}, \\
\dot{\boldsymbol{w}}_{1, R}=-\dot{w}_{1, R} \boldsymbol{e}_{R}, \dot{\boldsymbol{w}}_{2, R}=\dot{w}_{2, R} \boldsymbol{e}_{R} .
\end{gathered}
$$

It has been shown 1, 2, that the steady state conditions can be obtained from minimization of the generalized wear dissipation power for the case of wear of two bodies

$$
D_{w}^{(q)}=\sum_{i=1}^{2}\left(\int_{S_{c}}\left(\boldsymbol{t}_{i}^{c} \cdot \boldsymbol{w}_{i}\right)^{q} \mathrm{~d} S\right)^{1 / q}=\sum_{i=1}^{2} C_{i}^{1 / q},
$$


where $q$ is the control parameter, usually $q \geqslant 0$.

Assume that the contact pressure $p_{n}(\boldsymbol{x})$ and the friction shear stress satisfy the global equilibrium conditions for the body $B_{1}$, so we have

$$
\begin{aligned}
& \boldsymbol{f}=-\int_{S_{c}} \tilde{\boldsymbol{n}}_{c} p_{n} \mathrm{~d} S+\boldsymbol{f}_{0}=\mathbf{0}, \\
& \boldsymbol{m}=-\int_{S_{c}} \Delta \boldsymbol{r} \times \tilde{\boldsymbol{n}}_{c} p_{n} \mathrm{~d} S+\boldsymbol{m}_{0}=\mathbf{0},
\end{aligned}
$$

where $\boldsymbol{f}_{0}$ and $\boldsymbol{m}_{0}$ denote the resultant force and moment acting on the body $B_{1}, \Delta \boldsymbol{r}$ is the position vector.

Introducing the Lagrange multipliers $\dot{\boldsymbol{\lambda}}_{F}$ and $\dot{\boldsymbol{\lambda}}_{M}$, the equilibrium conditions in (1.9), we can state the Lagrangian functional

$$
L_{D_{w}}^{(q)}=L_{D_{w}}^{(q)}\left(p_{n}, \dot{\boldsymbol{\lambda}}_{F}, \dot{\boldsymbol{\lambda}}_{M}\right)=D_{w}^{(q)}\left(p_{n}\right)+(b+1) \dot{\boldsymbol{\lambda}}_{F} \cdot \boldsymbol{f}+(b+1) \dot{\boldsymbol{\lambda}}_{M} \cdot \boldsymbol{m}
$$

where it is assumed that $b=b_{1}=b_{2}[3$.

Satisfying the stationary condition of $1.10, \delta_{p_{n}} L_{D w}^{(q)}=0$, the contact pressure distribution is obtained in the form

$$
p_{n}=\left(\frac{\dot{\boldsymbol{\lambda}}_{F} \cdot \widetilde{\boldsymbol{n}}_{c}+\left(\dot{\boldsymbol{\lambda}}_{M} \times \Delta \boldsymbol{r}\right) \cdot \widetilde{\boldsymbol{n}}_{c}}{\left(\widetilde{\beta}_{1} v_{r}^{a_{1}}\right)^{q} C_{1}^{\frac{1-q}{q}}+\left(\widetilde{\beta}_{2} v_{r}^{a_{2}}\right)^{q} C_{2}^{\frac{1-q}{q}}}(1 \mp \mu \tan \chi)^{-q}\right)^{\frac{1}{(b+1) q-1}}
$$

and the equilibrium equations are

$$
\begin{aligned}
\boldsymbol{f}\left(\dot{\boldsymbol{\lambda}}_{F}, \dot{\boldsymbol{\lambda}}_{M}\right) & =-\int_{S_{c}} \tilde{\boldsymbol{n}}_{c} p_{n} \mathrm{~d} S+\boldsymbol{f}_{0}=\mathbf{0}, \\
\boldsymbol{m}\left(\dot{\boldsymbol{\lambda}}_{F}, \dot{\boldsymbol{\lambda}}_{M}\right) & =-\int_{S_{c}} \Delta \boldsymbol{r} \times \tilde{\boldsymbol{n}}_{c} p_{n} \mathrm{~d} S+\boldsymbol{m}_{0}=\mathbf{0} .
\end{aligned}
$$

Let us note that the orientation angle $\chi=\chi\left(\dot{\boldsymbol{\lambda}}_{F}, \dot{\boldsymbol{\lambda}}_{M}\right)$ depends on the Lagrange multiplier vectors and equations 1.12 are highly nonlinear. The Lagrange multiplier vectors $\dot{\boldsymbol{\lambda}}_{F}, \dot{\boldsymbol{\lambda}}_{M}$ can be calculated by applying the Newton-Raphson technique, as these variables are internal unknowns.

The control parameter $q$ provides the transition from local to global response. In fact, for $q=1$, these functionals provide the global measures but for $q \rightarrow \infty$ they represent local values of integrands. It has been shown that for $q=1$, the optimal solution corresponds to steady state condition. Thus, this condition can be specified directly from (1.11) and 1.12 instead of integration of the wear rule (1.1) for the whole transient wear process until the steady state is reached. We shall first illustrate the applicability of the stationary conditions to the analysis of several specific examples.

In the work of Goryacheva 8 we find same examples for cases when the wear parameters are not uniform along the contact surface. Such a situation occurs for inhomogeneous bodies. In [8] (Chapter 7), a case was analyzed when one of the bodies 
executes a rigid body translation displacement. The problem was solved analytically for special boundary conditions (contact surface of periodical character). We would like to analyze similar cases, when there is rigid body rotation and the contact surface is not only a plane. The heat generation in the contact zone is accounted for and the effect of temperature field on the steady wear state is analyzed. It is assumed that strains are small and the materials of the contacting bodies are linearly elastic. Our solution has been obtained by using the $p$-version finite element method [9, 10].

\section{WEAR ANALYSIS IN RING SEGMENT-ON-ROTATING DISK TESTS}

Consider first the interaction of stationary ring shoes with rotating disk of thickness $t_{\text {th }}$ and radius $R_{0}$ (Figure 2). Assume the plane stress state within the rings and disk.

The shoes are loaded uniformly by the pressure $\tilde{p}$ on the upper/lower boundary with the resulting force $F_{0}$ and may translate along the $z$-axis in order to arrange the interaction with disk at the contact surfaces specified by the angles $\pm \alpha_{0}$. The formulation will be for the upper shoe. In this case the rigid body wear velocity equals $\dot{\boldsymbol{\lambda}}_{F}=-\dot{\lambda}_{F} \boldsymbol{e}_{z}$. The disk is rotated with the angular velocity $\omega$ in the clockwise or anticlockwise direction.

Following our main assumption $(1.6)$, the wear rate vector is collinear with $\boldsymbol{e}_{z}$, thus

$$
\begin{aligned}
& \boldsymbol{e}_{R}=-\boldsymbol{e}_{z}, \quad \chi=\alpha, \\
& \boldsymbol{n}_{c}=-\cos \alpha \boldsymbol{e}_{z}-\sin \alpha \boldsymbol{e}_{x}, \quad \boldsymbol{e}=\boldsymbol{e}_{\tau 1}=\sin \alpha \boldsymbol{e}_{z}-\cos \alpha \boldsymbol{e}_{x}, \quad \boldsymbol{e}_{\tau 2}=\boldsymbol{e}_{y}, \\
& \widetilde{\boldsymbol{n}}_{c}=\boldsymbol{n}_{c} \pm \mu \boldsymbol{e}_{\tau},
\end{aligned}
$$

where the lower sign $(-)$ corresponds to the case of disk rotation in the anticlockwise direction and the upper sign $(+)$ to the rotation in the opposite direction. The contact pressure distribution resulting from the stationary condition 1.11 is expressed as follows:

$$
p_{n}=\left(\frac{\dot{\lambda}_{F}}{\sum_{i=1}^{2}\left(\widetilde{\beta}_{i} v_{r}^{a_{i}}\right)^{q} C_{i}^{\frac{1-q}{q}}}\right)^{\frac{1}{(b+1) q-1}}(\cos \alpha \mp \mu \sin \alpha)^{\frac{1-q}{(b+1) q-1}}(\cos \alpha)^{\frac{q}{(b+1) q-1}} .
$$

The equilibrium condition of the shoe is

$$
-\int_{-\alpha_{0}}^{\alpha_{0}} p_{n}\left(\boldsymbol{n}_{c} \pm \mu \boldsymbol{e}_{\tau}\right) \cdot \boldsymbol{e}_{z} R_{0} t_{\mathrm{th}} \mathrm{d} \alpha=F_{0}
$$

and provides the expression for the loading force

$$
\begin{array}{r}
F_{0}=\int_{-\alpha_{0}}^{\alpha_{0}}\left(\frac{\dot{\lambda}_{F}}{\sum_{i=1}^{2}\left(\widetilde{\beta}_{i} v_{r}^{a_{i}}\right)^{q} C_{i}^{\frac{1-q}{q}}}\right)^{\frac{1}{(b+1) q-1}} \\
(\cos \alpha \mp \mu \sin \alpha)^{\frac{1-q}{(b+1) q-1}+1}(\cos \alpha)^{\frac{q}{(b+1) q-1}} R_{0} t_{\mathrm{th}} \mathrm{d} \alpha .
\end{array}
$$




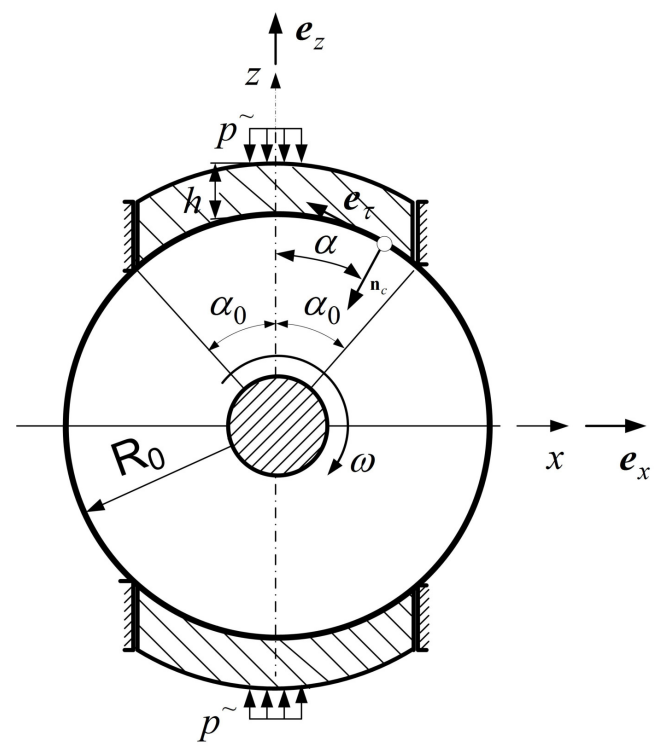

Figure 2. Ring-on-disk test, loading and geometrical parameters

Two cases will be discussed now.

Case A: Assume that $\widetilde{\beta}_{2}=0$, i.e., wear of the disk does not occur. Introducing the integral

$$
I_{D_{w}}^{\mp(q)}=\int_{-\alpha_{0}}^{\alpha_{0}} \frac{1}{\left(\widetilde{\beta}_{1} v_{r}^{a_{1}}\right)^{\frac{q}{(b+1) q-1}}}(\cos \alpha \mp \mu \sin \alpha)^{\frac{1-q}{(b+1) q-1}+1}(\cos \alpha)^{\frac{q}{(b+1) q-1}} R_{0} t_{\mathrm{th}} \mathrm{d} \alpha
$$

we obtain

$$
\frac{F_{0}}{I_{D_{w}}^{\mp(q)}}=\left(\frac{\dot{\lambda}_{F}}{C_{1}^{\frac{1-q}{q}}}\right)^{\frac{1}{(b+1) q-1}}
$$

and the contact pressure is expressed as follows:

$$
p_{n}=\frac{F_{0}}{I_{D_{w}}^{\mp(q)}}(\cos \alpha \mp \mu \sin \alpha)^{\frac{1-q}{(b+1) q-1}}\left(\frac{\cos \alpha}{\widetilde{\beta}_{1} v_{r}^{a_{1}}}\right)^{\frac{q}{(b+1) q-1}} .
$$

If the term $(b+1) q-1=0$ a singular pressure distribution occurs with localization at the centre line or perimeter line of contact zone. The steady wear state is reached for $q=1$ and then contact pressure distribution is

$$
p_{n}=\frac{F_{0}}{I_{D_{w}}^{\mp(q=1)}}\left(\frac{\cos \alpha}{\widetilde{\beta}_{1}\left(R_{0} \omega\right)^{a_{1}}}\right)^{\frac{1}{b}} .
$$


The wear rate component in the normal direction to the contact surface equals

$$
\dot{w}_{n}=\left(\widetilde{\beta}_{1}\left(R_{0} \omega\right)^{a_{1}}\right)\left(\frac{F_{0}}{I_{D_{w}}^{\mp(q=1)}}\right)^{b} \frac{\cos \alpha}{\widetilde{\beta}_{1}\left(R_{0} \omega\right)^{a_{1}}} \neq \text { const }
$$

and the wear rate along the vertical $z$-axis

$$
\dot{w}=\dot{w}_{R}=\dot{\lambda}_{F}=\frac{\dot{w}_{n}}{\cos \alpha}=\left(\frac{F_{0}}{I_{D_{w}}^{\mp(q=1)}}\right)^{b}=\text { const }
$$

is constant. The wear volume rate now equals

$$
\dot{W}=\int_{-\alpha_{0}}^{\alpha_{0}} \dot{w}_{n} R_{0} t_{\mathrm{th}} \mathrm{d} \alpha=\int_{-\alpha_{0}}^{\alpha_{0}} \dot{w}_{v} \cos \alpha R_{0} t_{\mathrm{th}} \mathrm{d} \alpha=\left(\frac{F_{0}}{I_{D_{w}}^{\mp(q=1)}}\right)^{b} R_{0} t_{\mathrm{th}} 2 \sin \alpha_{0} .
$$

Case B: At the end, we finalize our results for $q=1$ at $\widetilde{\beta}_{i}=\beta_{i} \mu^{b}$ and $v_{r}=\left\|\dot{\boldsymbol{u}}_{\tau}\right\|$. Then using (2.2) and 2.3) we have

$$
F_{0}=\int_{-\alpha_{0}}^{\alpha_{0}}\left(\frac{\dot{\lambda}_{F}}{\sum_{i=1}^{2}\left(\widetilde{\beta}_{i} v_{r}^{a_{i}}\right)}\right)^{\frac{1}{b}}(\cos \alpha \mp \mu \sin \alpha)(\cos \alpha)^{\frac{1}{b}} R_{0} t_{\mathrm{th}} \mathrm{d} \alpha
$$

and after introducing the integral

$$
\widetilde{I}_{D_{w}}^{\mp(q=1)}=\int_{-\alpha_{0}}^{\alpha_{0}}\left(\frac{1}{\sum_{i=1}^{2}\left(\widetilde{\beta}_{i} v_{r}^{a_{i}}\right)}\right)^{\frac{1}{b}}(\cos \alpha \mp \mu \sin \alpha)(\cos \alpha)^{\frac{1}{b}} R_{0} t_{\mathrm{th}} \mathrm{d} \alpha
$$

we obtain

$$
\frac{F_{0}}{\widetilde{I}_{D_{w}}^{\mp(q=1)}}=\left(\dot{\lambda}_{F}\right)^{\frac{1}{b}}
$$

so the contact pressure is expressed as follows:

$$
p_{n}=\frac{F_{0}}{\widetilde{I}_{D_{w}}^{\mp(q=1)}}\left(\frac{\cos \alpha}{\sum_{i=1}^{2}\left(\widetilde{\beta}_{i} v_{r}^{a_{i}}\right)}\right)^{\frac{1}{b}}, \quad v_{r}=R_{0} \omega
$$

The wear rate component in the contact normal direction equals

$$
\dot{w}_{n}=\sum_{i=1}^{2}\left(\widetilde{\beta}_{i} v_{r}^{a_{i}}\right)\left(\frac{F_{0}}{\widetilde{I}_{D_{w}^{+}(q=1)}}\right)^{b} \frac{\cos \alpha}{\sum_{i=1}^{2}\left(\widetilde{\beta}_{i} v_{r}^{a_{i}}\right)} \neq \text { const }
$$


and the wear rate along the $z$-axis according to $(2.9)$ is constant

$$
\dot{w}_{R}=\left(\frac{F_{0}}{\widetilde{I}_{D_{w}}^{\mp(q=1)}}\right)^{b}=\text { const. }
$$

The wear volume rate now equals

$$
\dot{W}=\int_{-\alpha_{0}}^{\alpha_{0}} \dot{w}_{n} R_{0} t_{\mathrm{th}} \mathrm{d} \alpha=\int_{-\alpha_{0}}^{\alpha_{0}} \dot{w}_{v} \cos \alpha R_{0} t_{\mathrm{th}} \mathrm{d} \alpha=\left(\frac{F_{0}}{\widetilde{I}_{D_{w}}^{\mp(q=1)}}\right)^{b} R_{0} t_{\mathrm{th}} 2 \sin \alpha_{0} .
$$

\section{Example 1}

Select the following specific parameter values: loading force $F_{0}=10 \mathrm{kN}$, contact angle $\alpha_{0}= \pm 30^{\circ}$, disk radius $R_{0}=200 \mathrm{~mm}$, and thickness $t_{\mathrm{th}}=10 \mathrm{~mm}$. The wear parameters are $\beta_{1}=0.0002, \beta_{2}=0, a=b=1$, the friction coefficient is $\mu=0.25$, the angular disk velocity $\omega=2.5 \mathrm{rad} / \mathrm{s}, \tilde{\beta}_{1}=\beta_{1} \mu, \widetilde{c}=\widetilde{\beta}_{1}\left(R_{0} \omega\right)=\widetilde{\beta}_{1} v_{r}, \widetilde{\beta}_{2} v_{r}=0$. The wear coefficient is equal to $\widetilde{c}=c 1=0.025$ in the intervals $-\alpha_{0} \leq \alpha \leq-15,15 \leq \alpha \leq \alpha_{0}$ and in the middle zone $\widetilde{c}=c 1 / 2$. The wear resistance in the middle zone is greater than in the exterior zones.

Minimizing the wear dissipation power $D_{w}^{(q)}\left(p_{n}\right)$, the contact pressure is specified by (2.6). Figures 3 presents the pressure distribution for anticlockwise and clockwise disk rotation. The pressure distribution depends on the orientation of disk rotation. If $q$ is close to 0.5 the contact pressure and wear distribution are highly localized at the contact zone perimeter or at the centre line.

Figures 4 presents the associated wear distribution for different values of $q$, the steady state wear process occurs for which $\dot{w}_{v}=\dot{w}_{R}=$ const and the pressure distribution is specified by (2.7). The steady state pressure distribution is symmetric with respect to the $z$-axis and does not depend on the friction coefficient.

\section{Example 2}

Consider now the loading shoe in the form of a thin ring segment of constant thickness $t_{\mathrm{th}}=10 \mathrm{~mm}$, subjected to the distributed load localized at the centre, so that the vertical traction is $p^{\sim}=49.5 \mathrm{MPa}$ and the resultant force $F_{0}=10 \mathrm{kN}$. Figure 5 presents the finite element mesh of the system and the initial contact pressure distribution with no gap (clockwise rotation). In the vertical direction there are 4 layers, along the long direction there are 11 elements. In the disk radial direction there are 4 elements, in the circumferential direction there are $15+11+15=41$ elements. For solution of the contact problem [11] the $p$-version finite element method 9] was used. The initial contact pressure distribution is highly localized near the centre line and due to friction on the right side boundary, (see Figure $5 \mathrm{p}$ ).

The steady state of wear is reached when the contact pressure distribution specified by (2.7) for $q=1$ is satisfied.

We would like consider the thermo-mechanical problem with angular velocity $\omega=$ $5 \mathrm{rad} / \mathrm{s}$. The friction heat is generated in the contact surface $S_{c} \in\left(r=200 \mathrm{~mm}, \alpha_{0}=\right.$ $\left.\mp 30^{\circ}\right)$. Friction coefficient is $\mu=0.25$. In the surface shoe $S_{q} \in(r=200 \mathrm{~mm}$, $x=\mp 100 \mathrm{~mm})$, in the surface of the $\operatorname{disc} S_{q} \in(r=200 \mathrm{~mm})$ the heat convection 

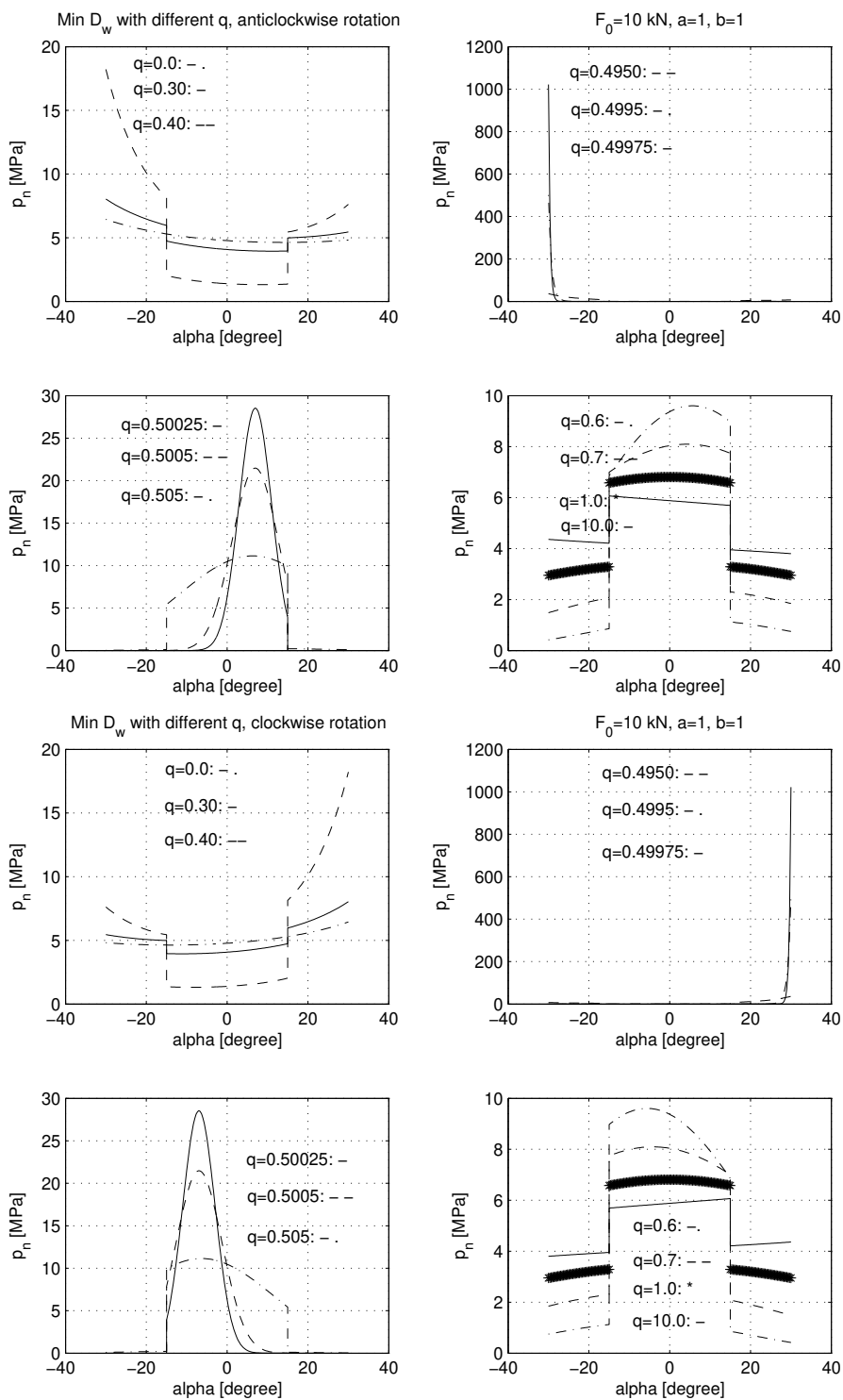

Figure 3. Contact pressure distribution for anticlockwise (rows 1,2), and clockwise (rows 3,4) disk rotation for different values of the control parameter $q$

condition is assumed, and in the surface $S_{\theta} \in(r=50 \mathrm{~mm})$ zero temperature is prescribed. The heat convection condition is also supposed in the whole plane surface. In view of anti-symmetry of the thermo-mechanical problem (we have two shoes) 

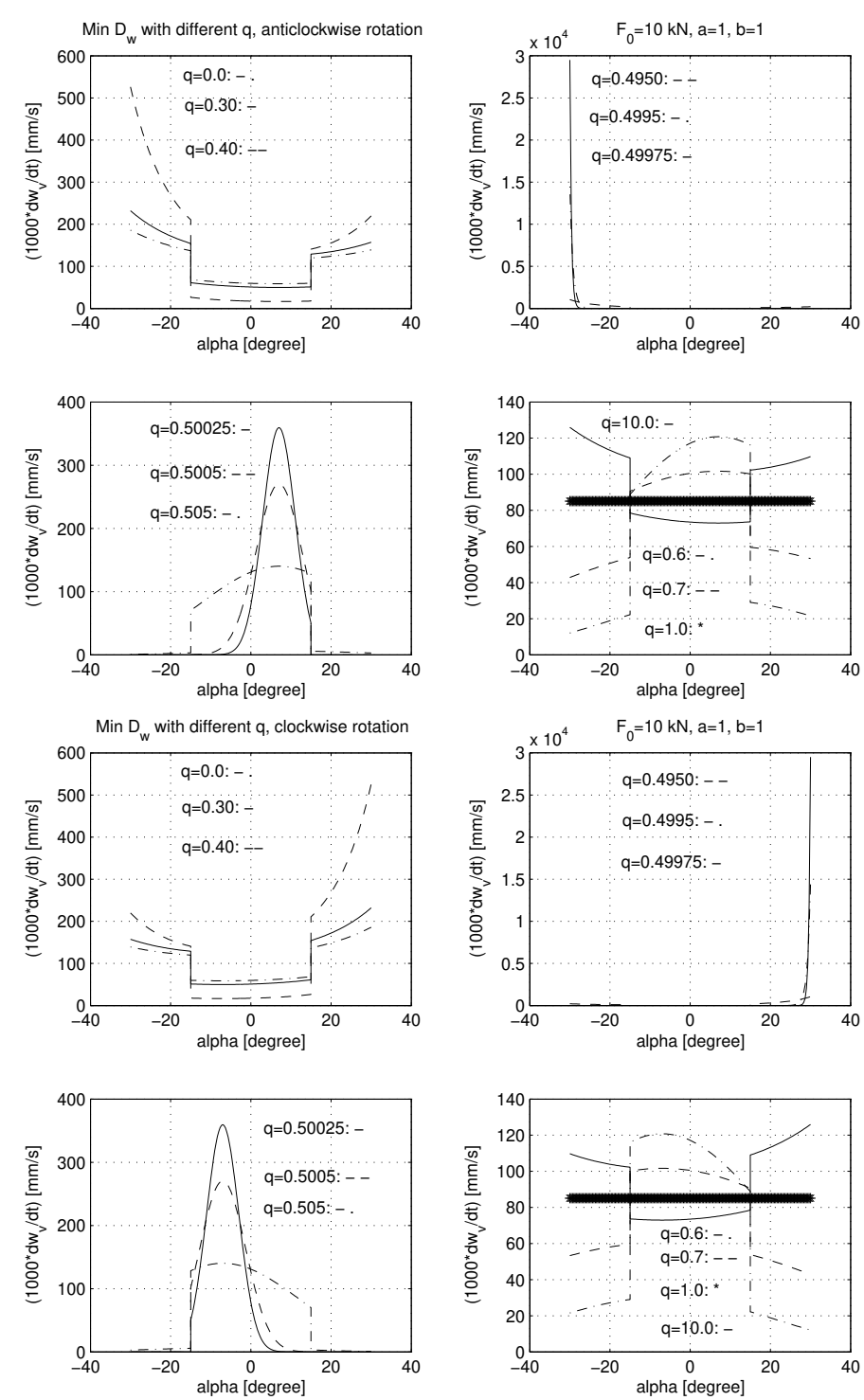

Figure 4. Vertical wear rate distribution for anticlockwise (rows 1,2), and clockwise (rows 3,4) disk rotations for different values of $q$

the thermo field must satisfy $\theta(x, z)=\theta(-x,-z)$ condition, that is in the axis $x$ is $\theta(x)=\theta(-x)$, and for displacement field is $\boldsymbol{u}(x)=\boldsymbol{u}(-x)$.

We shall analyze different shoe (Body 1) variants.

1. Material homogeneity for whole body 1, and wear parameters are uniform. 
2. Material homogeneity for whole body 1 and wear parameters are not uniform (see Example 1).

3. The shoe is made from different materials. In the finite element layer 1-2 in the interval material $-\alpha_{0} \leq \alpha \leq-15,15 \leq \alpha \leq \alpha_{0}$ is steel, in the middle part material is a composite (see Figure 5f). The composite segment is in the region: $-\alpha^{-} \leq \alpha \leq \alpha^{+}, R_{0} \leq r \leq R_{0}+h_{a}$. Material parameters are given in Table 1. The remaining parts of the shoe and disk are made of steel. For the distribution of wear parameters, see Example 1.

Table 1. Mechanical and thermal parameters of the two materials.

\begin{tabular}{|l|c|c|c|c|c|c|c|}
\cline { 2 - 8 } \multicolumn{1}{c|}{} & $\begin{array}{c}K^{(i)} \\
{[\mathrm{W} / \mathrm{mK}]}\end{array}$ & $\begin{array}{c}h_{c} \\
{\left[\mathrm{~W} / \mathrm{m}^{2} \mathrm{~K}\right]}\end{array}$ & $\begin{array}{c}c^{(i)} \\
{[\mathrm{J} / \mathrm{kgK}]}\end{array}$ & $\begin{array}{c}\alpha_{\theta} \cdot 10^{5} \\
{[1 / \mathrm{K}]}\end{array}$ & $\begin{array}{c}E \cdot 10^{-5} \\
{[\mathrm{MPa}]}\end{array}$ & $\nu^{(i)}$ & $\begin{array}{c}\rho^{(i)} \\
{\left[\mathrm{kg} / \mathrm{m}^{3}\right]}\end{array}$ \\
\hline Mat. 1 steel & 55 & 80 & 460 & 1 & 2 & 0.3 & 7800 \\
\hline Mat. 2 composite & 5 & 80 & 1200 & 3 & 1.3 & 0.25 & 846 \\
\hline
\end{tabular}

In the case of 1 and 2 calculations the material used was homogeneous (steel), but wear parameters in variant 2 were not uniform.

a)

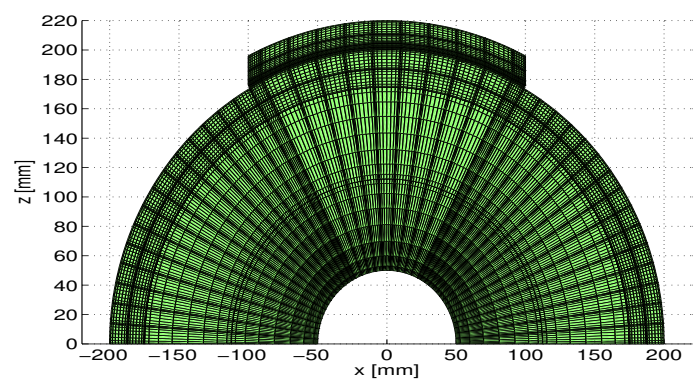

$F_{0}=10.0 \mathrm{kN}$, Original construction Contact pressure $[\mathrm{MPa}]$

b)

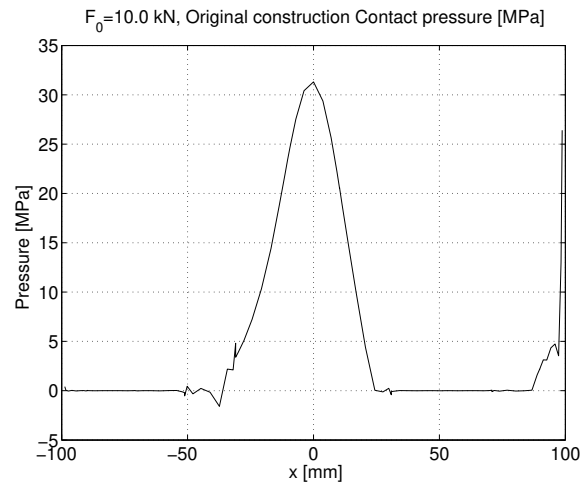

c)

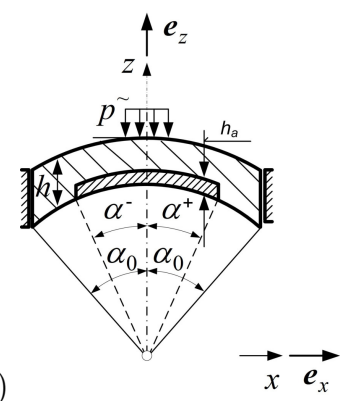

Figure 5. a) Finite element mesh for ring-disk system, b) initial contact pressure distribution (clockwise rotation), c) shoe geometry for Variant 3 
Using the $p$-version of the finite element technique for the solution of contact shape optimization problem (cf. Páczelt [1], Páczelt and Baksa 12], Páczelt and Mróz [1]), the stationary pressure distribution is reached for a small gap introduced with respect to the initial contact shape. The thermal problem is solved by using the upwinding Petrov-Galerkin formulation 2]. Using this technique the finite element solution does not exhibit oscillation. The weak variational formulation of mechanical and thermal problem can induce niggling oscillations, see 2, 4]. The finite element problems were solved by the $p$-version finite element technique using polynomial order $p=8$.
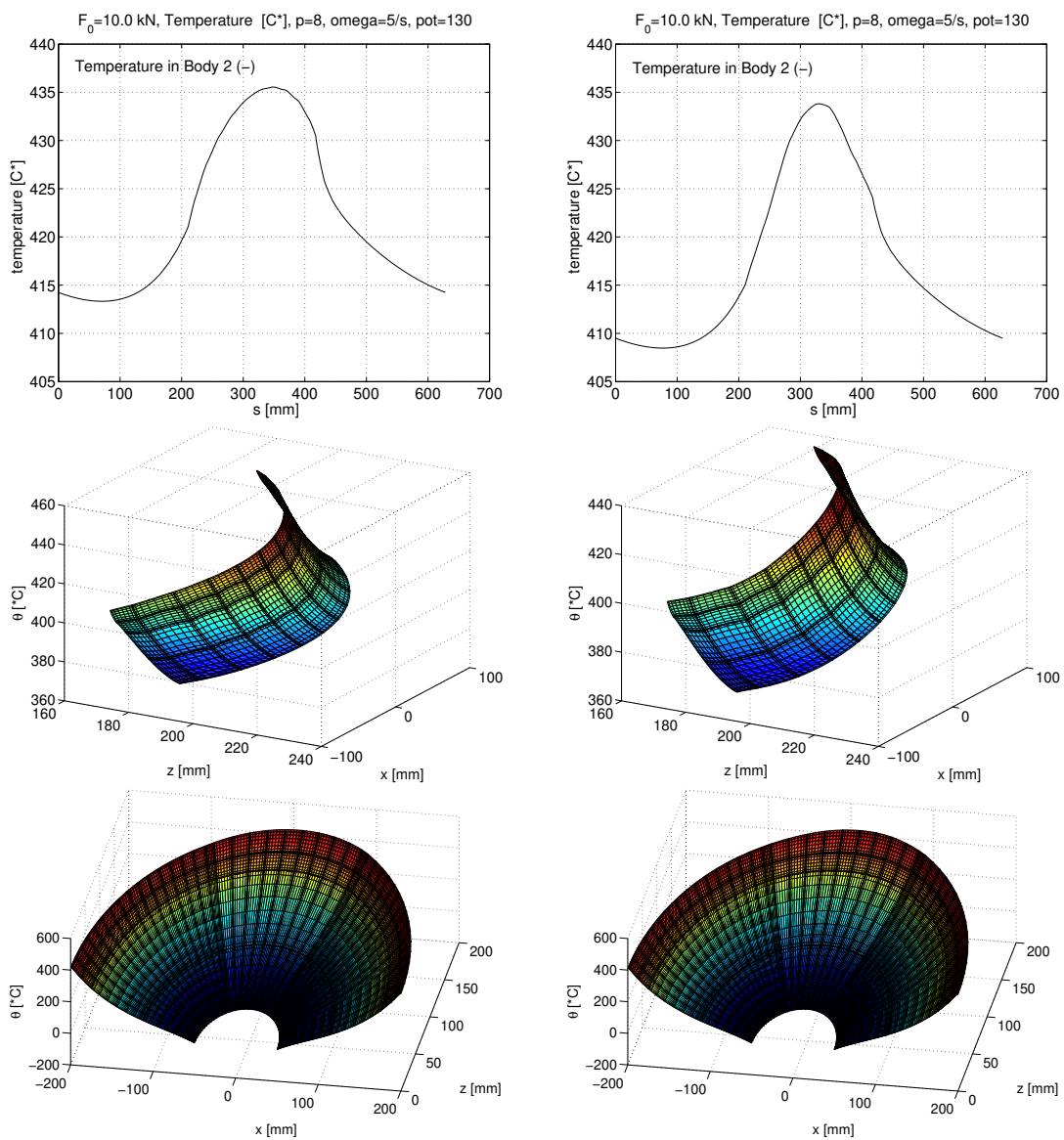

a)

b)

Figure 6. Temperature distribution in Body 2 along the radius $(r=$ $R_{0}=200 \mathrm{~mm}$ ) (top row), temperature field in the punch (middle row), temperature field in the disk for clockwise rotation at angular velocity $\omega=5 \mathrm{rad} / \mathrm{s}$ (bottom row) for a) Variant $1 \widetilde{\beta}=$ const, b) Variant $2 \widetilde{\beta} \neq$ const.

In Figure 6 the diagrams for Variants 1 and 2 can be seen. From top to bottom there are figures for temperature distribution on the disc boundary $\left(r=R_{0}=200 \mathrm{~mm}\right)$, 
inside the shoe, and inside the disc. Note that in Variant 2, the pressure is higher $\left(p_{n, \max }=6.887 \mathrm{MPa}\right)$ in the middle of the contact domain than in Variant $1\left(p_{n, \max }=\right.$ $5.286 \mathrm{MPa}$ ), where the line has a little pick form. Also we can see that at the boundary point $(r=200 \mathrm{~mm}, x=100 \mathrm{~mm})$ the temperature is higher than at the leading edge point $(r=200 \mathrm{~mm}, x=-100 \mathrm{~mm})$.

The total gap (shape) function is marked by the continuous line in Figure 7. The temperature distribution is marked by + , the gap without temperature effect is marked by $\circ$. The curves $(-)$ and (o) indicate that the effect of temperature distribution is significant. Heat flux distribution for Variant 1 and 2 is shown in Figure 8 . The difference between the two curves is very small.

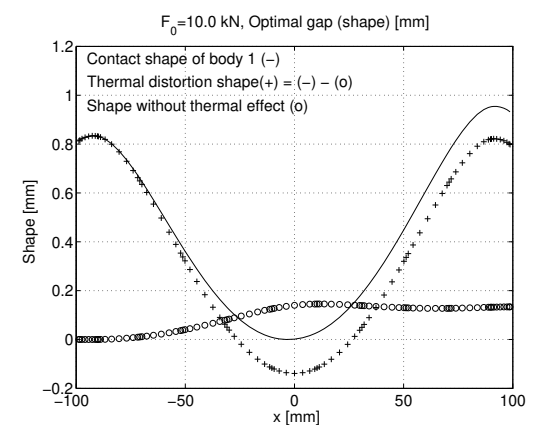

a)

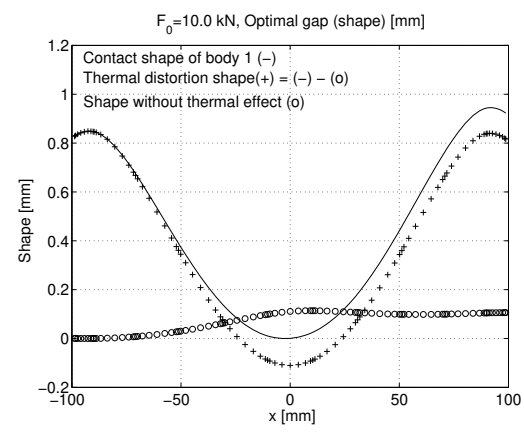

b)

Figure 7. Shape at steady wear state: a) Variant 1, b) Variant 2

Variant 3. Temperature distribution is shown in Figure 9. The optimal normal gap (Figure 9b) differs from gaps of Variant 2 (see Figure $7 \mathrm{~b}$ ). The elastic Young moduli are different in the central regions.

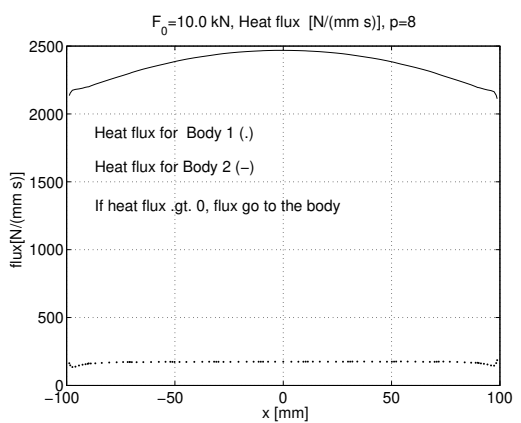

a)

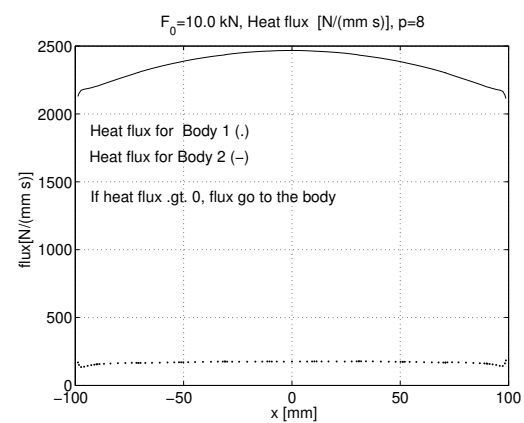

b)

Figure 8. Heat fluxes: (a) Variant 1, (b) Variant 2

It is seen in Figure 9ct that temperature is well isolated in the two layers, as in the central part of the region $x=0,205 \leq z \leq 220$ the temperature is $\sim 350^{\circ} \mathrm{C}$, 


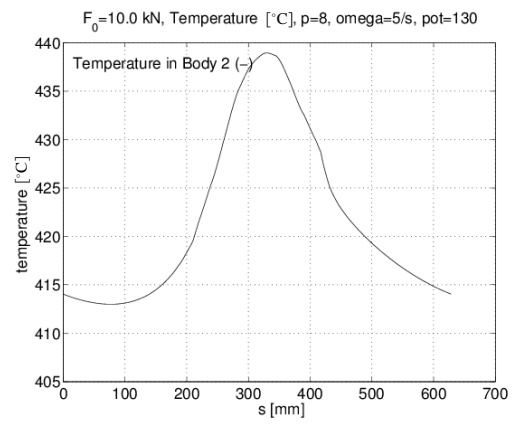

a)

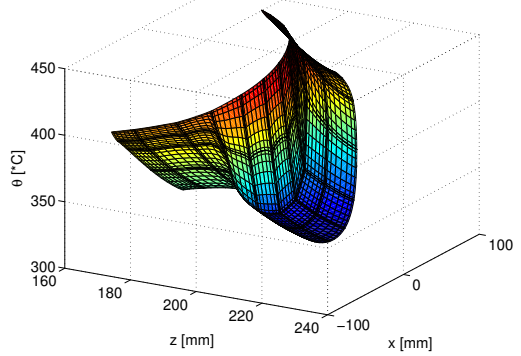

c)

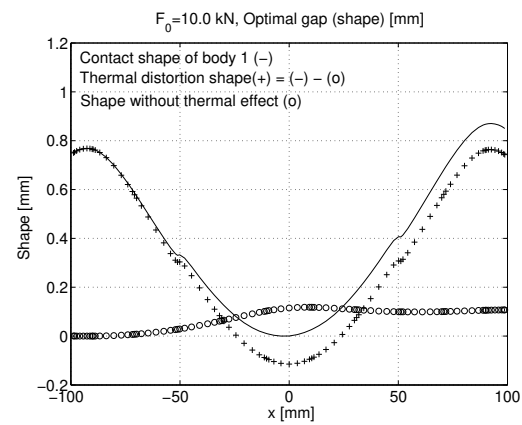

b)

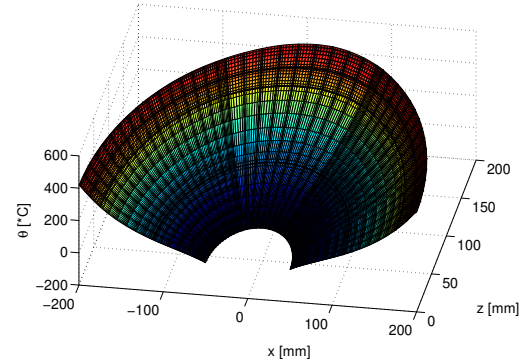

d)

Figure 9. a) Temperature distribution in the Body 2 along the radius $\left.\left(r=R_{0}=200 \mathrm{~mm}\right), \mathrm{b}\right)$ contact shape in the steady wear state, c) temperature field in the shoe, d) temperature field in the disk for clockwise rotation at angular velocity $\omega=5 \mathrm{rad} / \mathrm{s}$. Material of the shoe is inhomogeneous, problem: Variant 3

which approximately is lover by $\sim 90^{\circ} \mathrm{C}$ from the maximum $\sim 440^{\circ} \mathrm{C}$. The numerical analysis demonstrates that high temperature is also within the disc. In the steady state along the outer radius $\left(r=R_{0}=200 \mathrm{~mm}\right)$, the temperature varies by $\sim 25 \mathrm{C}^{\circ}$ in one rotation cycle.

One interesting point here is the heat flux partition between the shoe and the disc (see Figure 10). Because the disc rotates and its surface is larger than that of shoe, much heat is transferred from its body to the environment. As the conductivity coefficient is approximately 11 times smaller for material 2, in the center contact domain the heat flux is more oriented to the disc than to the shoe. The heat transfer ratio function has discontinuity in the interface of different materials.

\section{BLOCK UNDERGOING RIGID BODY TRANSLATION AND ROTATION}

Referring to Figure 11, consider a block (or punch) $B_{1}$ resting on a plane substrate $B_{2}$. It is loaded by the force $F_{0}$, also allowed to rotate around the pin $O$ and translate relative to the substrate along the direction $\widetilde{\boldsymbol{e}}_{x}$ normal to the contact plane $S_{c}$. The 


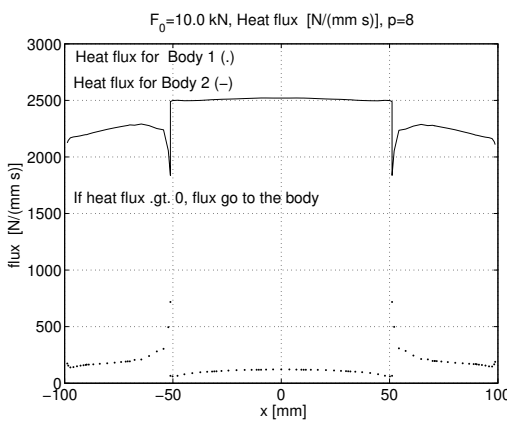

a)

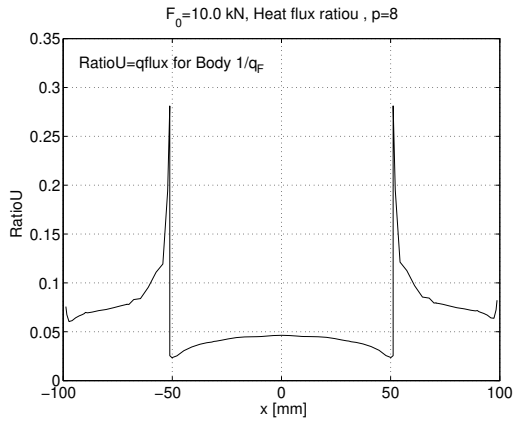

c)

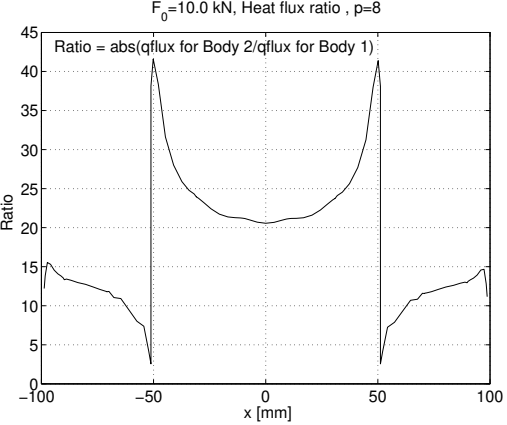

b)

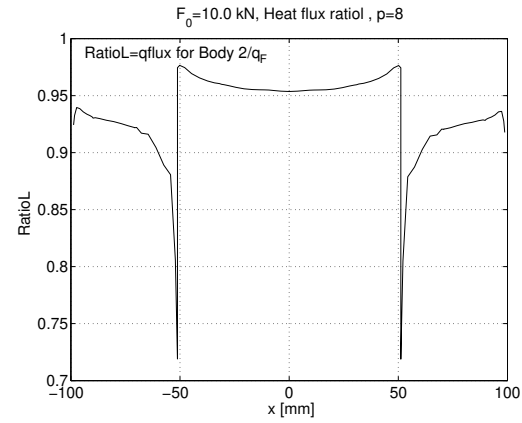

d)

Figure 10. a) Heat flux for Body 1 and 2,

b) Fluxratio: Ratio $=$ Flux $2 /$ Flux 1 ,

c) Flux ratio for the shoe: RatioU $=F \operatorname{Flux} 1 / q_{F},\left(q_{F}=\mu p_{n} R_{0} \omega\right)$,

d) Flux ratio for the disk: RatioL $=F l u x 2 / q_{F}$ at clockwise rotation at angular velocity $\omega=5 \mathrm{rad} / \mathrm{s}$. Material of the shoe is heterogeneous, problem: Variant 3

position of pin $O$ is fixed in the $(\widetilde{x}, \widetilde{z})$ reference system. The sliding velocity $v_{r}$ of substrate $B_{2}$ relative to $B_{1}$ can be oriented in the rightward or leftward directions. In the $\widetilde{x}, \widetilde{z}$ system the position of the pin $O$ is $(\widetilde{x}, \widetilde{z})=\left(l_{\widetilde{x}}, O\right)$. In our case the vertical wear velocity is $\dot{\boldsymbol{\lambda}}_{F}=-\dot{\lambda}_{F} \widetilde{\boldsymbol{e}}_{x}$, the angular velocity is $\dot{\boldsymbol{\lambda}}_{M}=-\dot{\lambda}_{M} \widetilde{\boldsymbol{e}}_{y}$, so the rigid body velocity of the point $P$ on the contact surface $S_{c}$ is oriented along the vector

$$
\begin{aligned}
& \boldsymbol{e}_{R}=\frac{-\left(\dot{\lambda}_{F} \widetilde{\boldsymbol{e}}_{x}+\dot{\lambda}_{M} \widetilde{\boldsymbol{e}}_{y} \times \Delta \boldsymbol{r}\right)}{H}=\frac{-\left(\dot{\lambda}_{F}+\dot{\lambda}_{M} \widetilde{z}\right) \widetilde{\boldsymbol{e}}_{x}-\dot{\lambda}_{M} l_{\widetilde{x}} \widetilde{\boldsymbol{e}}_{z}}{H}= \\
& =-\cos \alpha \widetilde{\boldsymbol{e}}_{x}-\sin \alpha \widetilde{\boldsymbol{e}}_{z},
\end{aligned}
$$

where

$$
H=\sqrt{\left(\dot{\lambda}_{F}+\dot{\lambda}_{M} \widetilde{z}\right)^{2}+\left(\dot{\lambda}_{M} l_{\widetilde{x}}\right)^{2}}
$$


and $\alpha$ is the angle between the rigid body velocity vector and the normal vector $\boldsymbol{n}_{c}$ to $S_{c}$. If there is no rotation, then $\alpha=\alpha_{\min }=0, \boldsymbol{e}_{R}=\boldsymbol{e}_{R, \lambda_{F}}=\boldsymbol{n}_{c}=-\widetilde{\boldsymbol{e}}_{x}$. If there is no translation allowed, then $\alpha=\alpha_{\max }, \boldsymbol{e}_{R}=\boldsymbol{e}_{R, \lambda_{M}} \sin \alpha=\frac{l_{\widetilde{x}}}{\sqrt{l_{\widetilde{x}}^{2}+\widetilde{z}^{2}}}=\sin \alpha_{\max }$.

Let us analyze the steady state wear process. We have

$$
\chi=\alpha, \quad \boldsymbol{n}_{c}=-\widetilde{\boldsymbol{e}}_{x}, \quad \boldsymbol{e}_{\tau}=\widetilde{\boldsymbol{e}}_{z}, \quad \widetilde{\boldsymbol{n}}_{c}=\boldsymbol{n}_{c} \pm \mu \boldsymbol{e}_{\tau}
$$

where \pm signs refer to rightward and leftward sliding velocities. The support condition of the block $B_{1}$ require the horizontal displacement of block to vanish at the pin $O$, thus $w_{O}=0$ at $\widetilde{z}=0, \widetilde{x}=l_{\widetilde{x}}$ and the vertical displacement at $O$ is specified from the contact conditions on $S_{c}$. The moment of force $F_{0}$ with respect to $O$ equals $\boldsymbol{m}_{0}=-F_{0} L_{F} \widetilde{\boldsymbol{e}}_{y}=-M_{0} \widetilde{\boldsymbol{e}}_{y}$ and is equilibrated by the moment of the interface contact tractions.

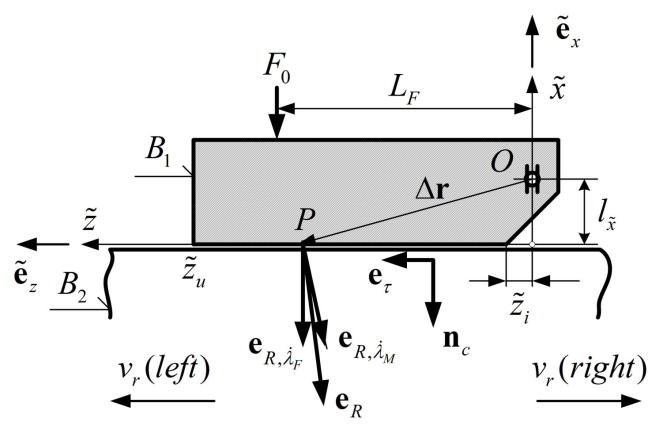

Figure 11. Rotating and translating block $B_{1}$ interacting with sliding body $B_{2}$

Starting from (1.11), the contact pressure is expressed as follows:

$$
\begin{aligned}
p_{n}=\left(\frac{\dot{\boldsymbol{\lambda}}_{F} \cdot \widetilde{\boldsymbol{n}}_{c}+\left(\dot{\boldsymbol{\lambda}}_{M} \times \Delta \boldsymbol{r}\right) \cdot \widetilde{\boldsymbol{n}}_{c}}{\left(\widetilde{\beta}_{1} v_{r}^{a_{1}}\right)^{q} C_{1}^{\frac{1-q}{q}}+\left(\widetilde{\beta}_{2} v_{r}^{a_{2}}\right)^{q} C_{2}^{\frac{1-q}{q}}}(1 \mp \mu \tan \chi)^{-q}\right)^{\frac{1}{c}}= \\
=\left(\frac{\dot{\lambda}_{F}+\dot{\lambda}_{M}\left(\widetilde{z} \mp \mu l_{\tilde{x}}\right)}{Z(q)}\right)^{\frac{1}{c}} Q(\alpha)^{-q / c},
\end{aligned}
$$

where $Z(q)=\left(\widetilde{\beta}_{1} v_{r}^{a_{1}}\right)^{q} C_{1}^{\frac{1-q}{q}}+\left(\widetilde{\beta}_{2} v_{r}^{a_{2}}\right)^{q} C_{2}^{\frac{1-q}{q}}, Q(\alpha)=(1 \mp \mu \tan \alpha), c=(b+1) q-1$.

The force and moment equilibrium conditions provide two equations:

$$
\begin{array}{r}
\int_{\widetilde{z}_{i}}^{\widetilde{z}_{u}}\left(\frac{\dot{\lambda}_{F}+\dot{\lambda}_{M}\left(\widetilde{z} \mp \mu l_{\tilde{x}}\right)}{Z(q)}\right)^{\frac{1}{c}} Q(\alpha)^{-q / c} t_{\mathrm{th}} \mathrm{d} \widetilde{z}=F_{0}, \\
\int_{\widetilde{z}_{i}}^{\widetilde{z}_{u}}\left(\frac{\dot{\lambda}_{F}+\lambda_{M}\left(\widetilde{z} \mp \mu l_{\tilde{x}}\right)}{Z(q)}\right)^{\frac{1}{c}} Q(\alpha)^{-q / c}\left(z \mp \mu l_{\tilde{x}}\right) t_{\mathrm{th}} \mathrm{d} \widetilde{z}=M_{0},
\end{array}
$$


where $t_{\mathrm{th}}$ is the block thickness in the lateral direction $y$. The $\mp$ signs refer to rightward and leftward sliding velocities. The non-linear equations (3.4) provide the values of Lagrange multipliers $\dot{\lambda}_{F}$ and $\dot{\lambda}_{M}$ specifying the rigid body translation and rotation wear velocities. They can be solved by applying the iterative Newton-Raphson technique.

The steady state regime occurs for $q=1$ and then we have $c=b, Z(q=1)=$ $\sum_{i=1}^{2} \widetilde{\beta}_{i} v_{r}^{a_{i}}$

In this case the contact pressure can be given in a simple form:

$$
p_{n}=\frac{\dot{\lambda}_{F}+\dot{\lambda}_{M} \widetilde{z}}{Z(q=1)}=\frac{\dot{\lambda}_{F}+\dot{\lambda}_{M} \widetilde{z}}{\sum_{i=1}^{2} \widetilde{\beta}_{i} v_{r}^{a_{i}}}
$$

hence, the pressure variation is a linear function of distance from the supporting pin.

\section{Example 3}

The bodies in contact are shown in Figure 12 with finite element mesh. The punch dimension in the horizontal direction is $L=60 \mathrm{~mm}$, its height is $h=100 \mathrm{~mm}$. The substrate strip is assumed to translate in the left direction, its measures: horizontal $0 \leq x \leq 1670$, vertical $0 \leq z \leq 100 \mathrm{~mm}$. The punch is loaded on the upper boundary $z=200 \mathrm{~mm}$ by the uniform pressure $p^{\sim}=16.66 \mathrm{MPa}$ corresponding to the resultant vertical force $F_{0}=10 \mathrm{kN}$. The thickness of punch and strip is $t_{\mathrm{th}}=10 \mathrm{~mm}$. The pin position is $l_{\widetilde{x}}=20 \mathrm{~mm}$. The punch motion in the vertical direction and rotation is associated with the wear velocity at the contact surface. The punch is assumed to be made of two materials. The lower punch portion of height $20 \mathrm{~mm}$ is characterized by the material parameters of Material 2, see Table1. The upper punch portion is

a)

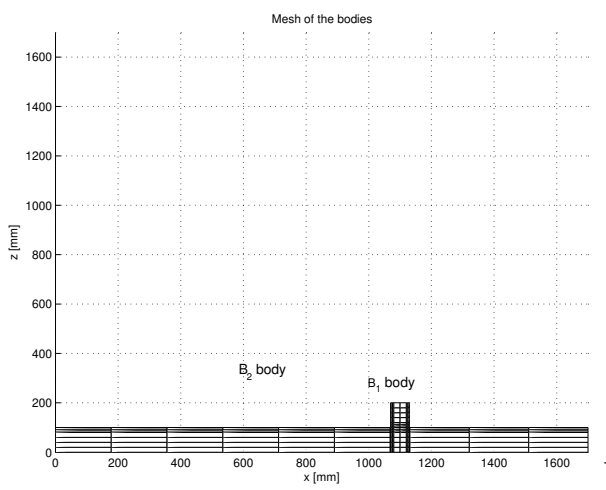

b)

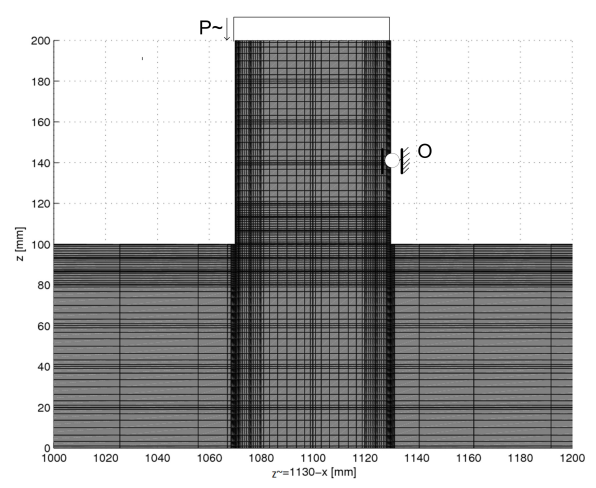

Figure 12. a) Finite element mesh, b) part of mesh for $p$-version finite element calculation. Load in the border $z=200$ is $p^{\sim}=16.66$ $\mathrm{MPa}$, pin is located at the point $x=1130 \mathrm{~mm}, z=140 \mathrm{~mm},(\widetilde{z}=$ $0, \widetilde{x}=40 \mathrm{~mm}$ ) 
assumed to be made of steel characterized by the parameters of Material 1. It is supposed that the ambient temperature $\theta_{a}$ of the surrounding medium is equal to zero. The coefficient of friction between bodies equals $\mu=0.25$. On the boundaries $z=0$ and $x=1670 \mathrm{~mm}$ the temperature is specified as $\theta_{a}=0$, on the other parts of the boundary $\left(x \in S_{q}^{(i)}, i=1,2\right)$ the heat flux is specified by the convection rule between the bodies and the surrounding medium. The contact pressure in the steady state is deduced from the principle of minimum of wear dissipation power defined by (3.5.

In order to specify the steady wear state, an infinite length model of strip should be used for calculation of the temperature field. In fact, for increasing sliding velocity the length of the strip should also be increased. For solution of this problem infinite elements must be used instead of adding new elements on the left of finite strip end [4]. The number of elements in $z$ direction is 7,8 elements in the $x$ direction in punch and in the strip at punch on the left and right side 11 and 4 elements.

Assuming $a=b=1, Z(q=1)=0.0002$, we can calculate the contact pressure distribution for right and leftward sliding directions.

In this example, problems for different variants of data will also be solved.

1. Variant 1. Wear parameters are uniform, strip is made from Material 1 and for punch material the parameters are characterized in the interval $100<z<120$ as Material 2, in the other part as Material 1.

2. Variant 2. Wear parameters are not uniform. In the part of punch $50 \leq \widetilde{z} \leq$ $60,0 \leq \widetilde{z} \leq 10$ there is $Z_{1}=Z(q=1)=0.0002$, and interval $10 \leq \widetilde{z} \leq 50$ there is $Z_{2}=Z(q=1)=0.0001$, but material parameters are the same as in Variant 1.

3. Variant 3. Wear parameters are as in Variant 2, and only in the interval $10 \leq \widetilde{z} \leq 50,0 \leq z \leq 20$ are material parameters for Material 2.

4. Variant 4. Material of all bodies is steel, and wear parameters are uniform, $Z=Z_{1}=$ const.

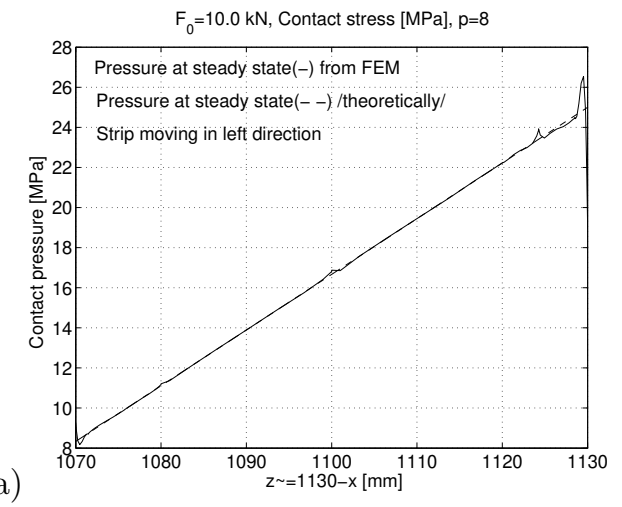

b)

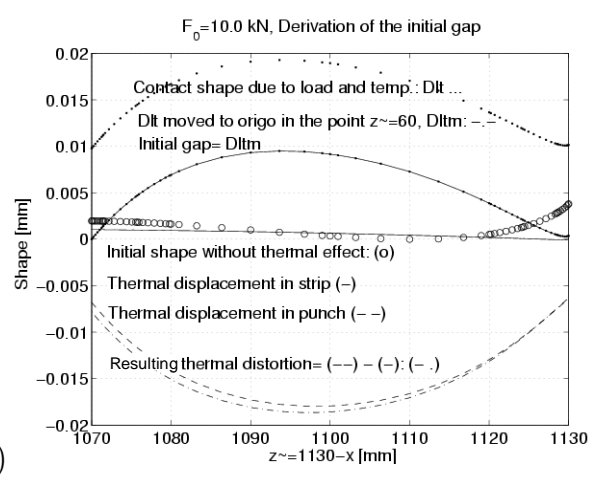

Figure 13. Variant 1: Solution of the const $\widetilde{\beta}_{1}$ for leftward sliding direction of the strip: a) contact pressure distribution, b) contact surface gap form in the steady wear state 
For Variant 1 the contact pressure is presented in Figure 13 , and the contact shape in Figure 13b. The difference between shapes specified neglecting (o) or accounting for temperature effect $(-\cdot-)$ is remarkable.

a)

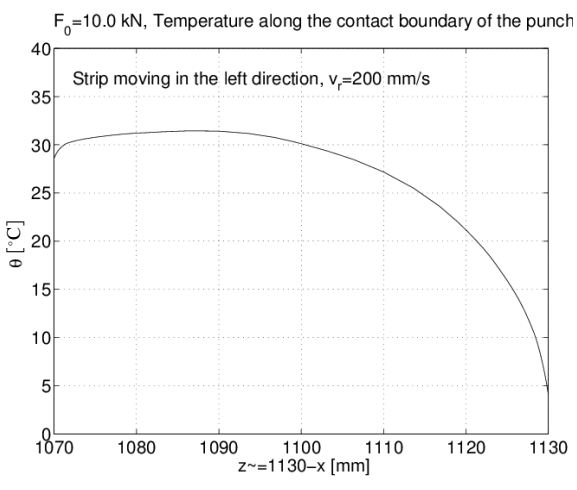

b)

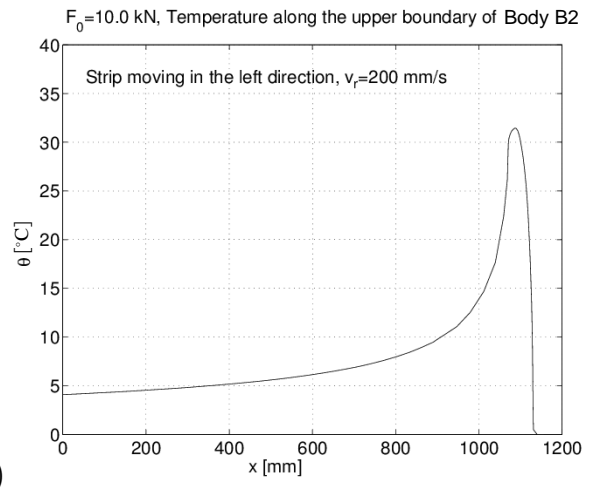

c)

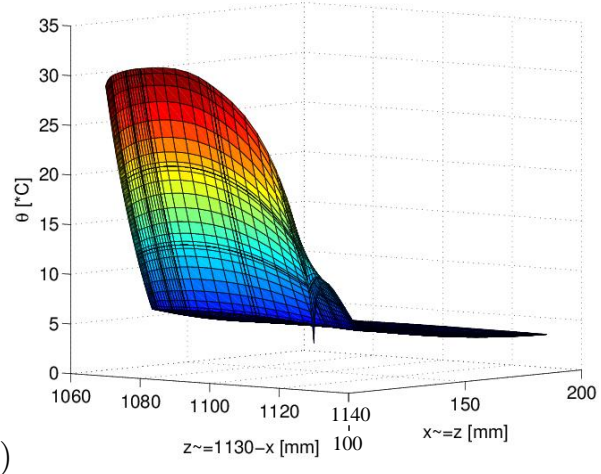

Figure 14. Variant 1: Temperature distribution for leftward sliding direction of the strip, a) in the shoe, b) in the strip, c) on the $x-z$ plane. The wear parameter is uniform.

The temperature distribution in the contact zone of shoe is shown in Figure $14 \mathrm{a}$, and in the strip interface $z=100 \mathrm{~mm}$ is in Figure 14 b. On the right side of the punch the temperature is practically zero, and on the left side far from punch position the temperature is very small. (The strip is moving in the left direction). The punch temperature on the left side is higher than that on the right side. The punch temperature also varies along direction $z$. It has a very different form for Variant 4 (see Figure 20b). In Variants 1-3 in the region of material 2 the gradient is very large, next it decreases to small values.

For Variant 4 the temperature variation is nearly constant. In other cases, material 2 provides a resistive barrier against the temperature transfer (see Figures 17, 19). Since upwinding Petrov-Galerkin technique was used for the numerical solution, the specified temperature field does not exhibit any oscillations. 

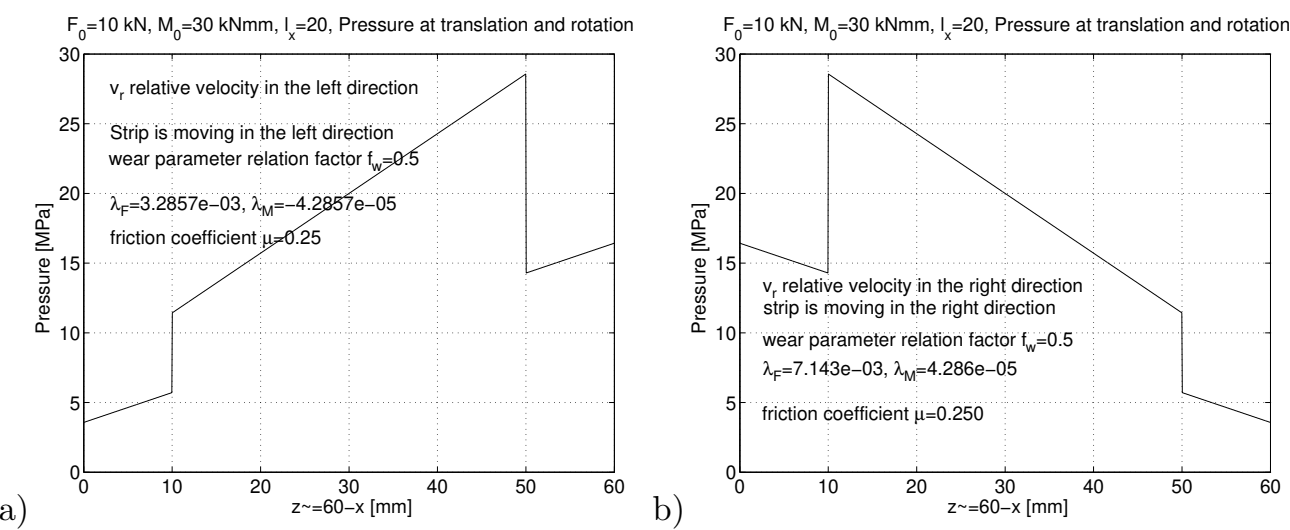

Figure 15. Variant 2,3: Contact pressure distribution for not uniform $\widetilde{\beta}_{1}$ : a) for leftward sliding direction of the strip, b) for rightward sliding direction of the strip, $f_{w}=Z_{2} / Z_{1}$
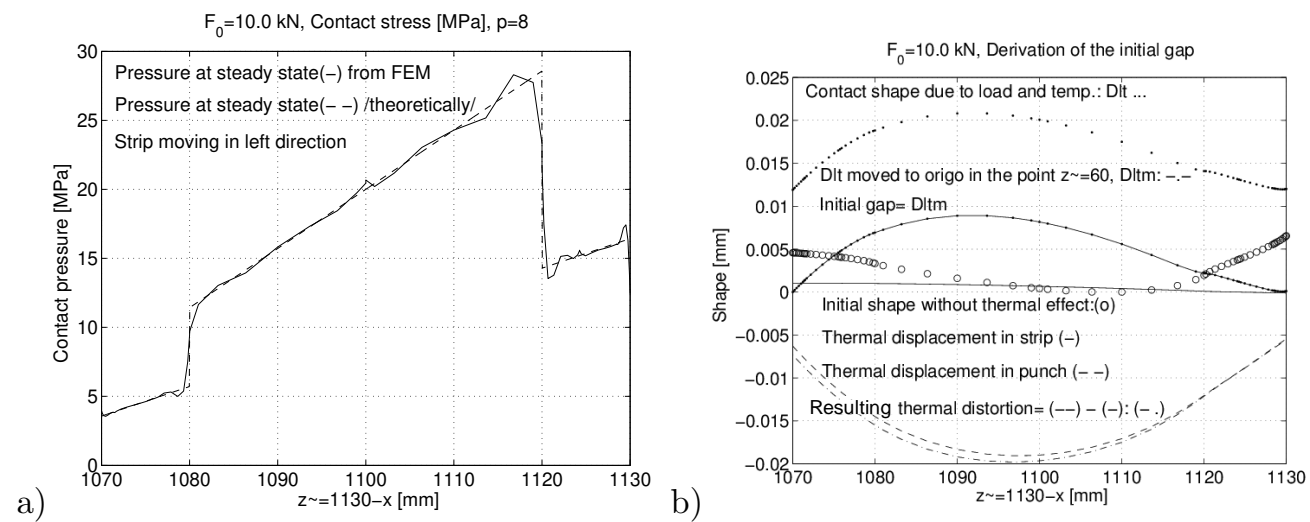

Figure 16. Variant 2: Solution of not uniform $\widetilde{\beta}_{1}$ for leftward sliding direction of the strip: a) contact pressure distribution, b) contact gap for steady wear state, $f_{w}=Z_{2} / Z_{1}=0.5$

In Figure 15 the theoretically determined distributions of contact pressure for Variants 2 and 3 are shown for different sliding directions of the strip. Figures 16 and 17 present the collected pressure, wear shape and temperature fields for Variant 2. The results for Variant 3 are collected in Figures 18 and 19 

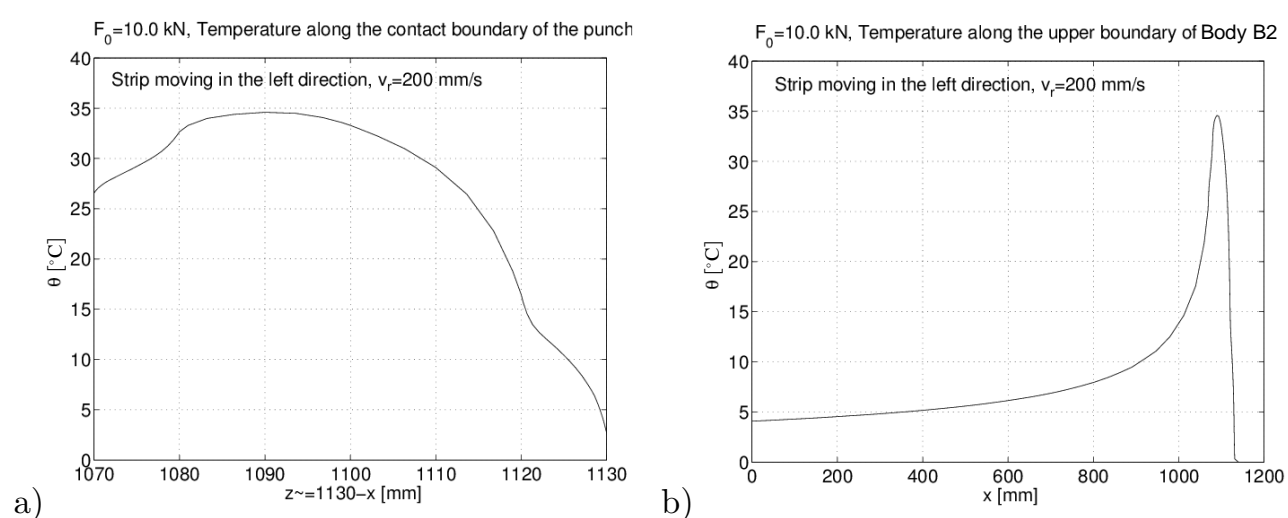

a)

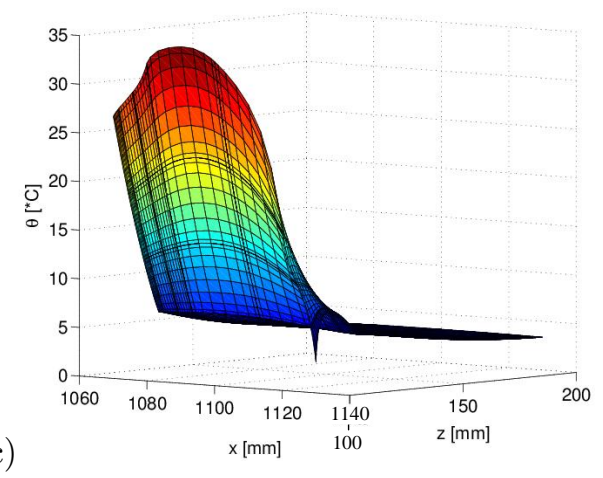

Figure 17. Variant 2: Temperature distribution for leftward sliding direction of the strip: a) in the shoe, b) in the strip, c) on the plane $x-z$. The wear parameter is non uniform, $f_{w}=Z_{2} / Z_{1}=0.5$.

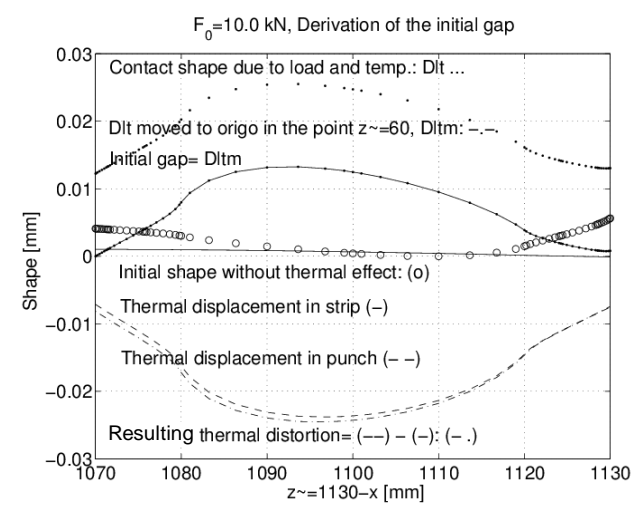

Figure 18. Variant 3: Solution for the non-uniform $\widetilde{\beta}_{1}$ and leftward sliding direction of the strip: contact gap form for steady wear state 
a)

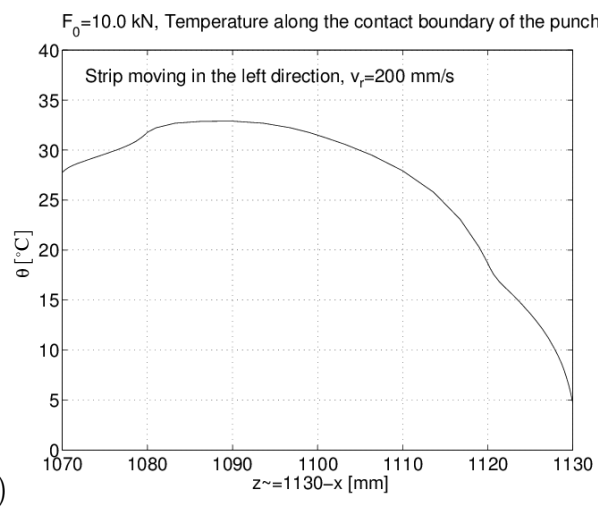

b)

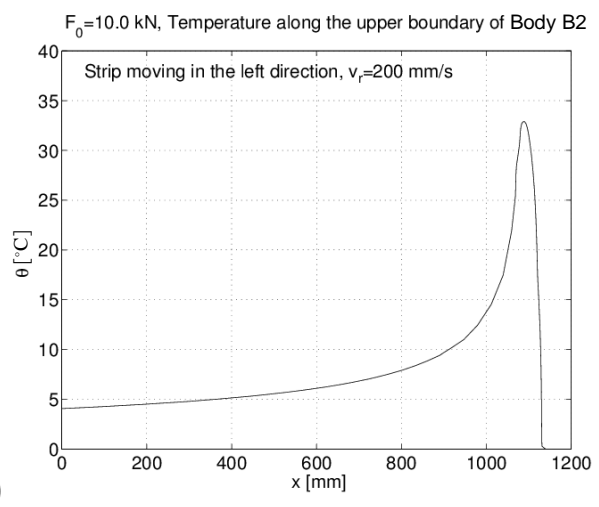

c)

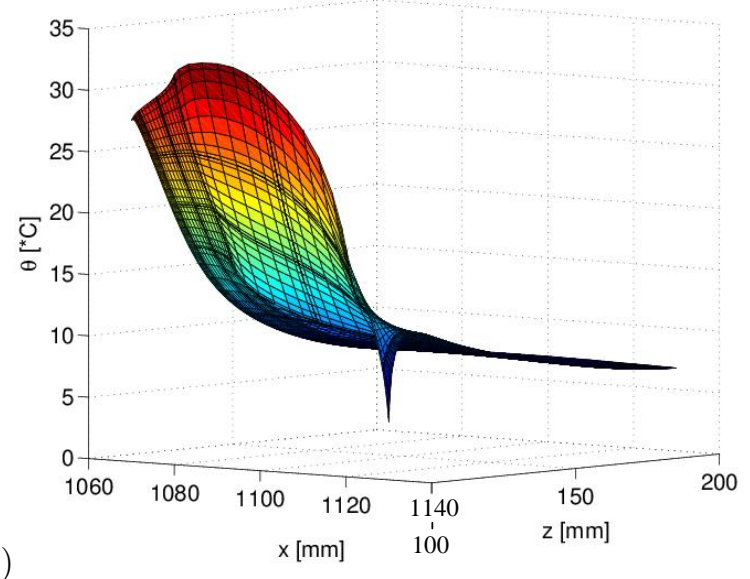

Figure 19. Variant 3: Temperature distribution for leftward sliding direction of the strip: a) in the shoe, b) in the strip, c) in the plane $x-z$. The wear parameter is not uniform, $f_{w}=Z_{2} / Z_{1}=0.5$.

Comparing results of Variants 2 and 3, it is noted that the temperature at the center of contact zone is a little bit larger for Variant 2 since then on both sides of the contact zone the material is steel and its coefficient of conductivity is larger. The initial gap at steady wear state is larger for Variant 3, since then the temperature distortions smaller relative to Variant 2 (see Figure 16 b, Figure 18). Certainly, for the Variant 4 design (steel is used for all elements) the temperature is lowest relative to the other variants (see Figure 20. 
a)

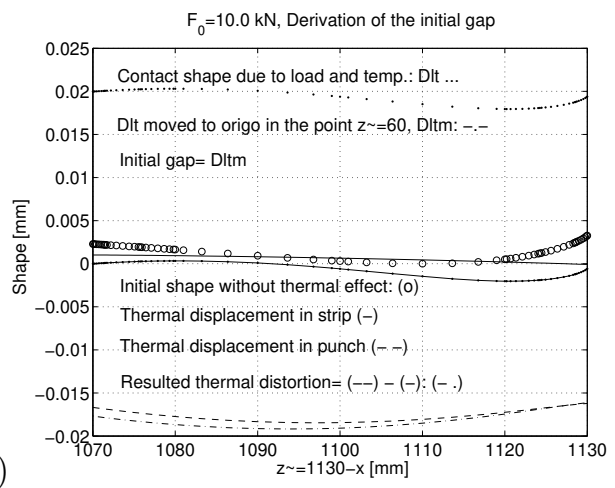

b)

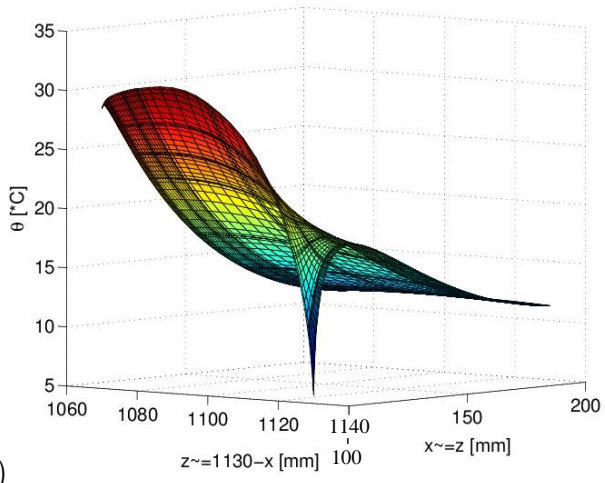

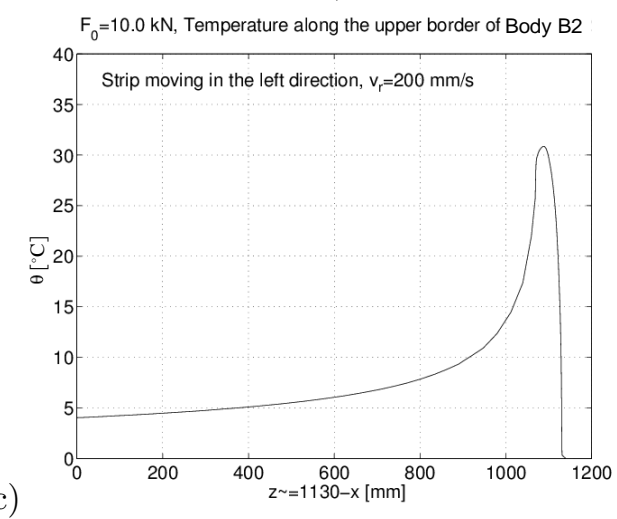

Figure 20. Variant 4: Material: steel, wear parameters are homogeneous. a) contact gap shape: b) temperature in the shoe (in the plane $x-z)$, c) temperature in the strip

\section{PERIOdiC SLIDING: WEAR DisSipation IN PERIODIC MOTION AND MAIN PRESSURE IN A PERIODIC STEADY WEAR STATE}

Consider now the periodic sliding motion, so that the relative displacement satisfies the condition

$$
u_{\tau}(t)=u\left(t+T_{*}\right)
$$

where $T_{*}$ is the period of sliding oscillation. In the steady wear state, the elastic stress and strain will vary periodically, thus

$$
\varepsilon(t)=\varepsilon\left(t+T_{*}\right), \sigma(t)=\sigma\left(t+T_{*}\right) .
$$

The numerical treatments of oscillating sliding motion by Peigney [13 and Kim et. al. 14 and the analytical investigation of Goryacheva et. al. 15 indicate the existence of steady states attained in the wear process.

In this section we shall analyze the wear process induced by the reciprocal strip translation, assuming $\widetilde{\beta}_{1} \neq 0, \widetilde{\beta}_{2}=0$, i.e. accounting only for punch wear. 
In 5 the wear parameter $\widetilde{\beta}_{1}$ was supposed to be uniform in the contact zone. In the present analysis it is assumed that the wear parameter varies along the sliding $x$ coordinate. We investigate these problems without friction heat generation in the contact surface. The heat generation was taken into account in 16 at uniform wear parameters.

During the steady periodic response the wear increment accumulated during one cycle should be compatible at each point $x \in S_{c}$ with the rigid body punch motion.

In the analysis the contact pressure distribution is assumed to be fixed during a semi-cycle and varies discontinuously during sliding reversal in consecutive semi-cycles [5].

The wear dissipation work for periodic motion is

$$
E_{w}=\frac{1}{2} \sum_{i=1}^{2} \int_{0}^{T_{*} / 2}\left(\int_{S_{c}^{(i)}} \boldsymbol{t}_{i}^{c+} \cdot \dot{\boldsymbol{w}}_{i}^{+} \mathrm{d} S\right) \mathrm{d} \tau+\frac{1}{2} \sum_{i=1}^{2} \int_{T_{*} / 2}^{T_{*}}\left(\int_{S_{c}^{(i)}} \boldsymbol{t}_{i}^{c-} \cdot \dot{\boldsymbol{w}}_{i}^{-} \mathrm{d} S\right) \mathrm{d} \tau
$$

where $\boldsymbol{t}_{i}^{c+}, \boldsymbol{t}_{i}^{c-}$ are the contact traction vectors and $\dot{\boldsymbol{w}}_{i}^{+}, \dot{\boldsymbol{w}}_{i}^{-}$are the wear velocities of the $i^{t h}$ body in the progressive and reciprocal motion direction, $T_{*}$ is the period of sliding motion, $T_{*}=2 \pi / \omega$ and $\omega$ is the angular velocity.

The strip slides periodically in the horizontal direction, thus we can write

$$
\boldsymbol{u}_{\tau}=-u_{0} \cos \omega \tau \boldsymbol{e}_{x}=u_{0} \cos \omega \tau \boldsymbol{e}_{\widetilde{z}}
$$

In our case the relative tangential velocity is (see Figure 11):

$$
\boldsymbol{u}_{\tau}=\dot{\boldsymbol{u}}_{\tau}^{(2)}-\dot{\boldsymbol{u}}_{\tau}^{(1)}=-u_{0} \omega \sin \omega \tau \boldsymbol{e}_{\widetilde{z}}=-u_{0} \omega \sin \omega \tau \boldsymbol{e}_{\tau}=-v_{r} \boldsymbol{e}_{\tau}
$$

with the corresponding shear stress acting on the body $B_{1}$ along $-\boldsymbol{e}_{\tau}$ The integral over one period of the relative velocity between the bodies is

$$
\int_{0}^{T_{*} / 2} v_{r} \mathrm{~d} \tau=\int_{T_{*} / 2}^{T_{*}} v_{r} \mathrm{~d} \tau=2 u_{0}
$$

In view of the wear rule (1.1) the wear dissipation for the punch of Figure 12 is

$$
E_{w}=\frac{1}{2} \int_{0}^{T_{*} / 2}\left(\int_{S_{c}^{(1)}} p_{n}^{+} \dot{w}_{1, n}^{+} \mathrm{d} S\right) \mathrm{d} \tau+\frac{1}{2} \int_{T_{*} / 2}^{T_{*}}\left(\int_{S_{c}^{(1)}} p_{n}^{-} \dot{w}_{1, n}^{-} \mathrm{d} S\right) \mathrm{d} \tau
$$

and for $\widetilde{\beta}_{1} \neq 0, \widetilde{\beta}_{2}=0, a_{1}=b_{1}=1$ there is

$$
\frac{E_{w}}{2 u_{0}}=\int_{S_{c}^{(1)}} \widetilde{\beta}_{1}\left\{\left(p_{n}^{+}\right)^{2}+\left(p_{n}^{-}\right)^{2}\right\} \mathrm{d} S=\frac{E_{w}^{+}}{2 u_{0}}+\frac{E_{w}^{-}}{2 u_{0}} .
$$

In the steady wear state $E_{w}$ reaches a minimum value. Let us note that $p_{n}^{+}$and $p_{n}^{-}$are not uniformly distributed on the contact interface. Take the coordinate $\widetilde{z}=$ $1130-x$. 
Assume the rigid body wear velocities for left $(-)$ and right $(+)$ directions of the substrate sliding motion in the following form:

$$
\dot{\lambda}_{F}^{-}=-\dot{\lambda}_{F}^{-} \boldsymbol{e}_{z}, \dot{\lambda}_{M}^{-}=\dot{\lambda}_{M}^{-} \boldsymbol{e}_{y}, \dot{\lambda}_{F}^{+}=-\dot{\lambda}_{F}^{+} \boldsymbol{e}_{z}, \dot{\lambda}_{M}^{+}=-\dot{\lambda}_{M}^{+} \boldsymbol{e}_{y}
$$

Thus the velocities at an arbitrary point of punch are

$$
-\left(\dot{\lambda}_{F}^{-}-\dot{\lambda}_{M}^{-} \widetilde{z}\right) \boldsymbol{e}_{z}, \quad \text { or } \quad-\left(\dot{\lambda}_{F}^{+}+\dot{\lambda}_{M}^{+} \widetilde{z}\right) \boldsymbol{e}_{z}
$$

After time integration there is $\Delta \lambda_{F, M}^{+}=\int_{0}^{T_{*} / 2} \dot{\lambda}_{F, M}^{+} \mathrm{d} \tau, \Delta \lambda_{F, M}^{-}=\int_{T_{*} / 2}^{T_{*}} \dot{\lambda}_{F, M}^{-} \mathrm{d} \tau$ and the displacements resulting from these velocities are

$$
-\left(\Delta \lambda_{F}^{+}+\Delta \lambda_{M}^{+} \widetilde{z}\right) \boldsymbol{e}_{z} \quad \text { and } \quad-\left(\Delta \lambda_{F}^{-+}+\Delta \lambda_{M}^{-} \widetilde{z}\right) \boldsymbol{e}_{z}
$$

Thus, the total wear accumulated during one sliding cycle is

$$
\Delta w_{n}=\Delta w_{n}^{+}+\Delta w_{n}^{-}=\left(\Delta \lambda_{F}^{+}+\Delta \lambda_{F}^{-}\right)+\left(\Delta \lambda_{M}^{+}-\Delta \lambda_{M}^{-}\right) \widetilde{z} .
$$

This value of the wear can be calculated from the wear law supposing $\widetilde{\beta}_{1} \neq 0$, $\widetilde{\beta}_{2}=0$ and $a_{1}=b_{1}=1$,

$$
\Delta w_{n}=\Delta w_{n}^{+}+\Delta w_{n}^{-}=U \widetilde{\beta}_{1}\left(p_{n}^{+}+p_{n}^{-}\right)=U \widetilde{\beta}_{1} p_{\Sigma}=2 U \widetilde{\beta}_{1} p_{m}
$$

where $p_{m}=\left(p_{n}^{+}+p_{n}^{-}\right) / 2$ is the main cyclic pressure, $p_{\Sigma}=\left(p_{n}^{+}+p_{n}^{-}\right)=2 p_{m}$ is the sum of contact pressure, $U=\int_{0}^{T_{*} / 2} v_{r} \mathrm{~d} \tau=2 u_{0}$.

Comparing 4.12, 4.13), it is seen that the distribution of the modified mean contact pressure values of consecutive semi-cycles must be a linear function of position, thus

$$
p_{\bmod \Sigma}(\widetilde{z})=\widetilde{\beta}_{1}(\widetilde{z}) p_{\Sigma}(\widetilde{z})=\widetilde{\beta}_{1}(\widetilde{z}) 2 p_{m}(\widetilde{z})=\widetilde{\beta}_{1}(\widetilde{z}) 2\left(p_{m}^{C}+p_{m}^{L} \widetilde{z}\right)
$$

i.e.

$$
\begin{array}{r}
\Delta w_{n}=\Delta w_{n}(\widetilde{z})=\Delta w_{n}^{+}+\Delta w_{n}^{-}=\left(\Delta \lambda_{F}^{+}+\Delta \lambda_{F}^{-}\right)+\left(\Delta \lambda_{M}^{+}-\Delta \lambda_{M}^{-}\right) \widetilde{z}= \\
=\widetilde{\beta}_{1}(z) \int_{0}^{T_{*} / 2}\left\|\dot{\boldsymbol{u}}_{\tau}\right\| \mathrm{d} \tau 2\left(p_{m}^{C}+p_{m}^{L} \widetilde{z}\right)=U \widetilde{\beta}_{1}(\widetilde{z}) 2\left(p_{m}^{C}+p_{m}^{L} \widetilde{z}\right)= \\
=U \widetilde{\beta}_{1}(\widetilde{z}) p_{\Sigma}(\widetilde{z})=U p_{\bmod \Sigma}(\widetilde{z}),
\end{array}
$$

where $\Delta \lambda_{F, M}^{ \pm}$is the increment of rigid body wear velocities in the half period time.

Using the equilibrium equations for summed loads, the summed pressure for the steady wear state is determined for $\widetilde{\beta}_{1}=$ const as

$$
\begin{aligned}
& p_{m}^{C}=\frac{F_{0}}{S_{c}}-\frac{3 F_{0}\left(-L+2 \widetilde{z}_{F}\right)}{L S_{c}}, p_{m}^{L}=\frac{6 F_{0}\left(-L+2 \widetilde{z}_{F}\right)}{L^{2} S_{c}}, \\
& p_{\Sigma}=2 p_{m}=p_{n}^{+}+p_{n}^{-}=2\left(p_{m}^{C}+p_{m}^{L} \widetilde{z}\right)
\end{aligned}
$$


where $\widetilde{z}_{F}$ is the coordinate of the resultant load $F_{0}=F_{0}\left(p^{\tilde{}}\right)$. For non-negativity of $p_{m}$ there should be $L / 2 \leq \widetilde{z}_{F} \leq 2 L / 3$. Here $S_{c}$ is the area of contact zone.

For $\widetilde{\beta}_{1} \neq$ const it turns out that the summed contact pressure is linear but notcontinuous function (see Figure 15 , but the modified function $p_{\bmod \Sigma}(\widetilde{z})=\widetilde{\beta}_{1}(\widetilde{z}) p_{\Sigma}(\widetilde{z})$ is linear and continuous.

The equilibrium equations are

$$
F_{0}=t_{\mathrm{th}} \int_{0}^{L} p_{m}(\widetilde{z}) \mathrm{d} \widetilde{z}, \quad M_{0}=t_{\mathrm{th}} \int_{0}^{L} \widetilde{z} p_{m}(\widetilde{z}) \mathrm{d} \widetilde{z} .
$$

In view of Figure 21, where the distribution of mean cyclic contact pressure is presented, we can write the following algebraic equation system for calculation of the values $p_{m}^{C}, p_{m}^{L}$

$$
\left[\begin{array}{ll}
A_{11} & A_{12} \\
A_{21} & A_{22}
\end{array}\right]\left[\begin{array}{l}
p_{m}^{C} \\
p_{m}^{L}
\end{array}\right]=\frac{1}{t_{\mathrm{th}}}\left[\begin{array}{c}
F_{0} \\
M_{0}
\end{array}\right]
$$

where

$$
\begin{aligned}
& A_{11}=\widetilde{z}_{1}+\frac{\widetilde{\beta}_{s}}{\widetilde{\beta}_{m}}\left(\widetilde{z}_{4}-\widetilde{z}_{1}\right)+\widetilde{z}_{5}-\widetilde{z}_{4}, \\
& A_{12}=A_{21}=\frac{1}{2}\left[\widetilde{z}_{1}^{2}+\frac{\widetilde{\beta}_{s}}{\widetilde{\beta}_{m}}\left(\widetilde{z}_{4}^{2}-\widetilde{z}_{1}^{2}\right)+\widetilde{z}_{5}^{2}-\widetilde{z}_{4}^{2}\right] \text {, } \\
& A_{22}=\frac{1}{3}\left[\widetilde{z}_{1}^{3}+\frac{\widetilde{\beta}_{s}}{\widetilde{\beta}_{m}}\left(\widetilde{z}_{4}-\widetilde{z}_{1}\right)\left(\widetilde{z}_{1}^{2}+\widetilde{z}_{4}^{2}+\widetilde{z}_{1} \widetilde{z}_{4}\right)+\left(z_{5}-z_{4}\right)\left(\widetilde{z}_{4}^{2}+\widetilde{z}_{5}^{2}+\widetilde{z}_{4} \widetilde{z}_{5}\right)\right] \text {. }
\end{aligned}
$$

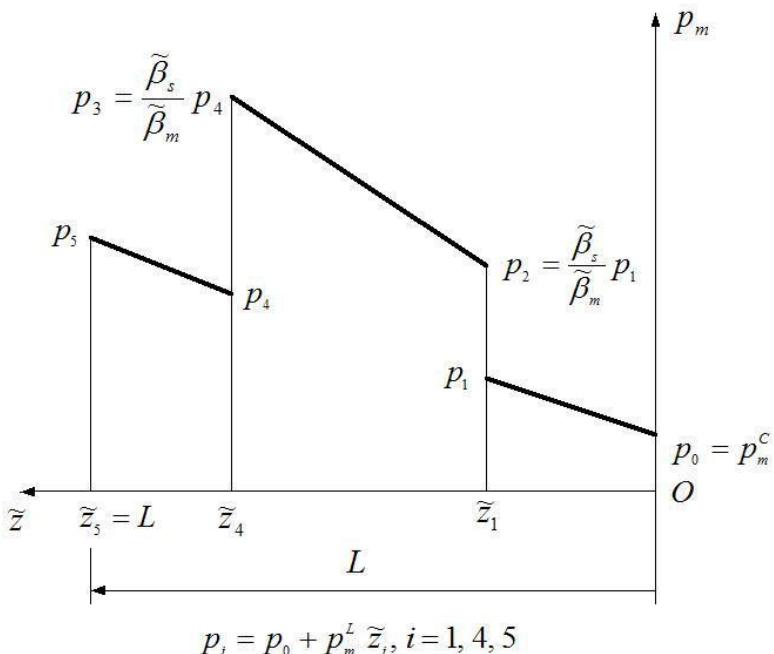

Figure 21. Distribution of the main cyclic pressure, $\left(\widetilde{\beta}_{s} / \widetilde{\beta}_{m}=2\right)$ 
Here $\widetilde{\beta}_{s}$ is the wear parameter for interval $0 \leq \widetilde{z} \leq \widetilde{z}_{1}$ and $\widetilde{z}_{4} \leq \widetilde{z} \leq \widetilde{z}_{5}$. In the interval $\widetilde{z}_{1} \leq \widetilde{z} \leq \widetilde{z}_{4}$ the wear parameter is $\widetilde{\beta}_{m}$.

The wear increment in one period (as was mentioned the contact pressure is fixed in half period)

$$
\Delta w_{n}=\widetilde{\beta}_{1}\left[p_{n}^{+}+p_{n}^{-}\right]\left(u_{0} \omega\right) \int_{0}^{T_{*} / 2}|\sin \omega \tau| \mathrm{d} \tau=\tilde{\beta}_{1} 2 p_{m} 2 u_{0},
$$

which using 4.16) provides the simple relation

$$
\Delta w_{n}=\Delta w_{n}(\widetilde{z})=2 U \widetilde{\beta}_{1}\left(p_{m}^{C}+p_{m}^{L} \widetilde{z}\right)=U \widetilde{\beta}_{1}(\widetilde{z}) p_{\Sigma}(\widetilde{z})=U p_{\bmod \Sigma}(\widetilde{z}) .
$$

The averaged wear rate in one period

$$
\dot{\bar{w}}_{n}=\frac{\Delta w_{n}}{T_{*}}=\widetilde{\beta}_{1} \frac{U\left(p_{n}^{+}+p_{n}^{-}\right)}{T_{*}}=\frac{U p_{\bmod \Sigma}}{T_{*}} .
$$

If the rigid body wear velocity $\lambda_{M}^{+}=\lambda_{M}^{-}=0$, then in the steady periodic wear regime the uniform wear increment is accumulated during the full cycle at each point of the contact zone and the following condition should be satisfied:

$$
\widetilde{\beta}_{1}(\widetilde{z})\left(p_{n}^{+}(\widetilde{z})+p_{n}^{-}(\widetilde{z})\right)=\widetilde{\beta}_{1}(\widetilde{z}) 2 p_{m}(\widetilde{z})=\widetilde{\beta}_{1}(\widetilde{z}) p_{\Sigma}(\widetilde{z})=p_{\bmod \Sigma}(\widetilde{z})=\text { const } .
$$

The steps of time integration of the wear rate rule can be found in 5].

\section{Example 4}

Let us analyze the wear of the punch (Body 1) shown in Figure 12 The following geometric parameters are assumed: the punch width is $L=60 \mathrm{~mm}$, its height is $h=100 \mathrm{~mm}$, the thickness of punch and strip is $t_{\mathrm{th}}=10 \mathrm{~mm}$.

The wear parameters are: $\widetilde{\beta}_{1}=1.25 \pi \cdot 10^{-5}, \widetilde{\beta}_{2}=0, a=b=1$, the coefficient of friction is $\mu=0.25$. The horizontal displacement of the substrate is $u_{\tau}=-u_{0} \cos \omega \tau$, where $u_{0}=1.5 \mathrm{~mm}, \omega=10 \mathrm{rad} / \mathrm{s}, \tau$ is the time. The material parameters are presented in Table 1 .

The upper parts of the punch and strip are assumed to be made of the same material (Material 1, see Table 1). The lower punch portion of height $20 \mathrm{~mm}$ is characterized by the parameters of Material 2, see Table 1

4.1. Symmetric load (Load case 1). Consider a symmetric punch loading on the upper boundary $z=200 \mathrm{~mm}$ at the uniform pressure $p^{\sim}=16.66 \mathrm{MPa}$ corresponding to the resultant vertical force $F_{0}=10 \mathrm{kN}$.

In the intervals $50 \leq \widetilde{z} \leq 60,0 \leq \widetilde{z} \leq 10$ the wear parameter $\widetilde{\beta}_{10}=\widetilde{\beta}_{1}$, and interval $10 \leq \widetilde{z} \leq 50, \widetilde{\beta}_{1 m}=\widetilde{\beta}_{1} / 2$.

Then the modified summa of contact pressure is

$$
p_{\text {mod }}(\widetilde{z})=\frac{\beta_{1}(\widetilde{z}) p_{\Sigma}(\widetilde{z})}{\widetilde{\beta}_{10}} \quad \text { if } \quad 50 \leq \widetilde{z} \leq 60, \quad 0 \leq \widetilde{z} \leq 10
$$

and

$$
p_{\text {mod }}(\widetilde{z})=\frac{\beta_{1}(\widetilde{z}) p_{\Sigma}(\widetilde{z})}{\widetilde{\beta}_{1 m}} \quad \text { if } \quad 10 \leq \widetilde{z} \leq 50
$$


a)

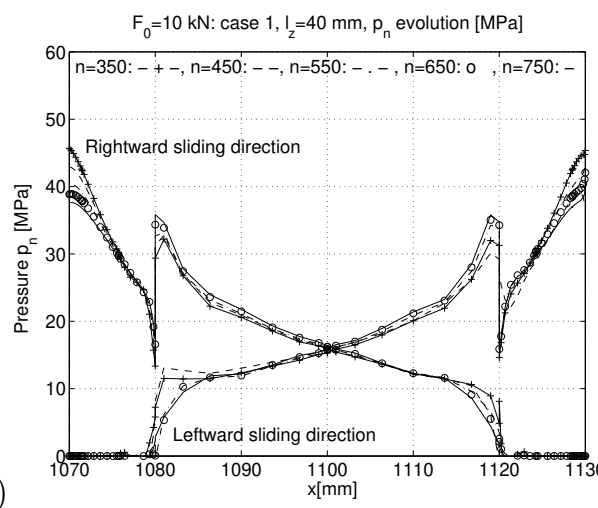

b)

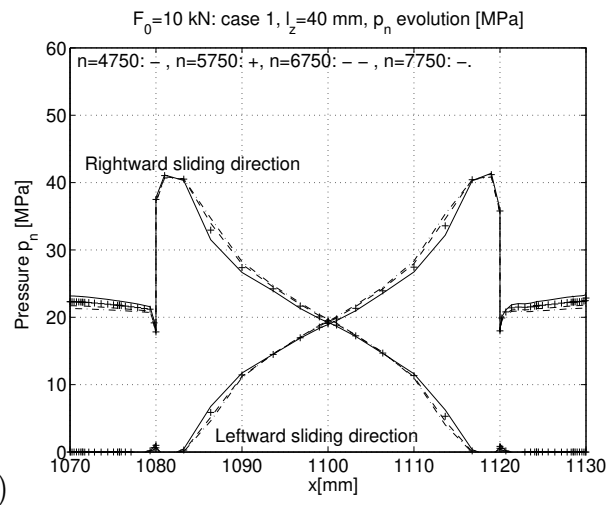

Figure 22. Contact pressure at different time steps

a)
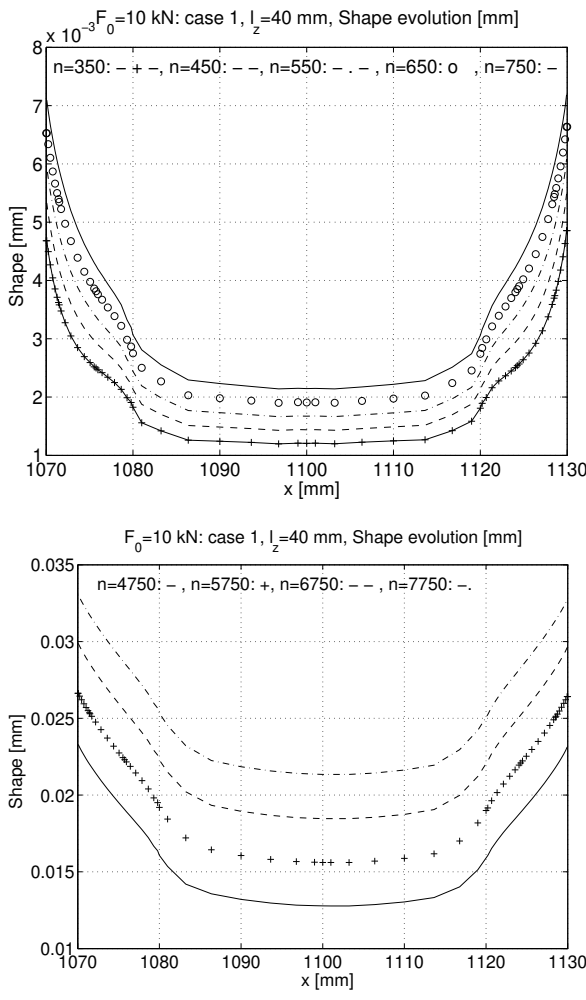

b)

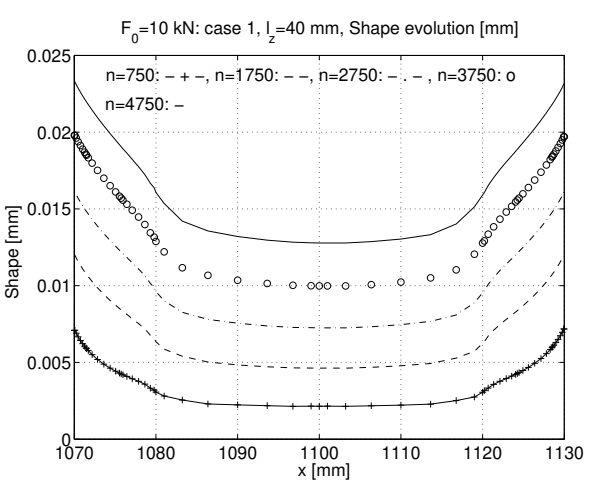

d)

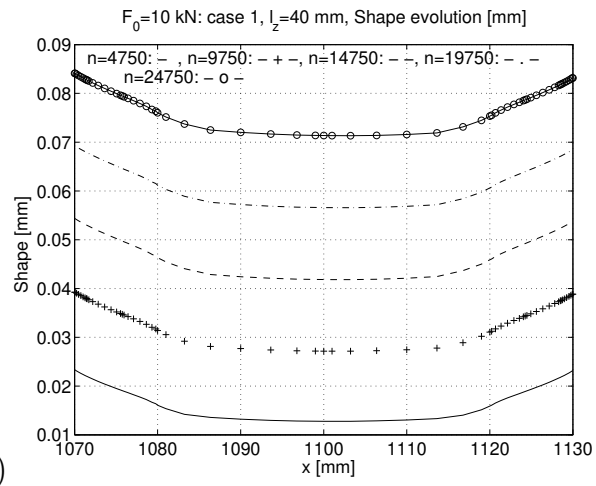

Figure 23. Shape evolution in the wear process 
a)

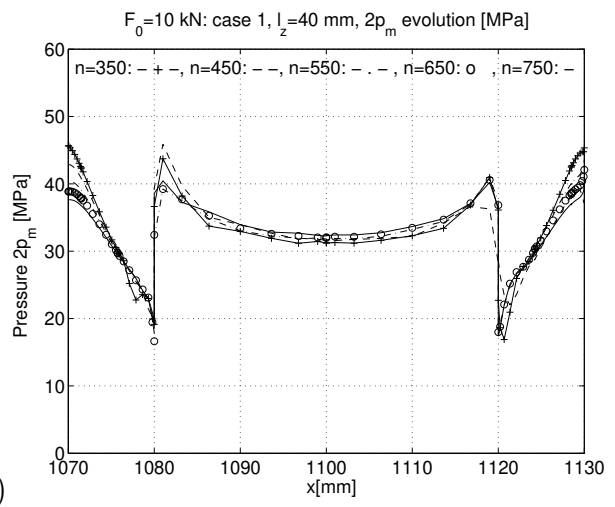

b)

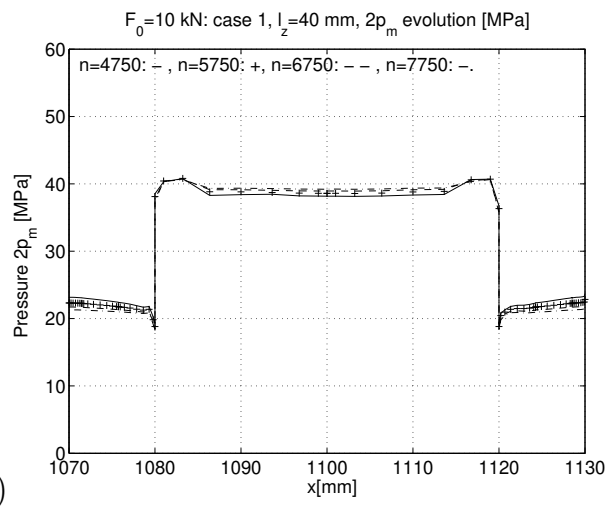

c)

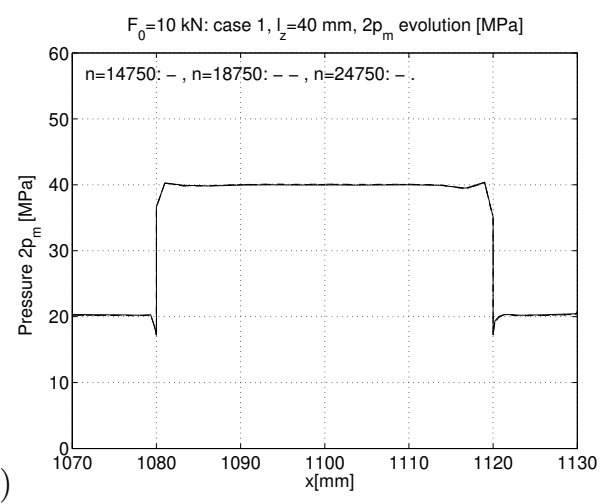

Figure 24. Summa of contact pressures in one cycle

In our case the modified summa of contact pressure is $p_{\bmod }(\tilde{z})=20 \mathrm{MPa}$. In fact, we have from 4.18 $\left(p_{m}^{C}=p_{0} / 2\right)$

$$
2 p^{\sim} L=2 p^{\sim} 60=p_{0} 10+p_{0} 40 \cdot 2+p_{0} 10=p_{0} 100, \quad p_{0}=1.2 p^{\sim}=p_{\bmod }(\widetilde{z}) .
$$

In the steady wear state this relation must be preserved in each period. In this case, Body 1 translates in the vertical direction at the constant velocity $\dot{\lambda}_{F}$. The numerical solution follows this theoretical result (see Figures 22 25). Here $n$ is the number of half periods. It is clear that after $n \geq 14000$ the steady cyclic wear state is reached, the functions of sum of contact pressures (Figure 24) and the modified sum of contact pressures (Figure 25) are fixed in time. 
a)
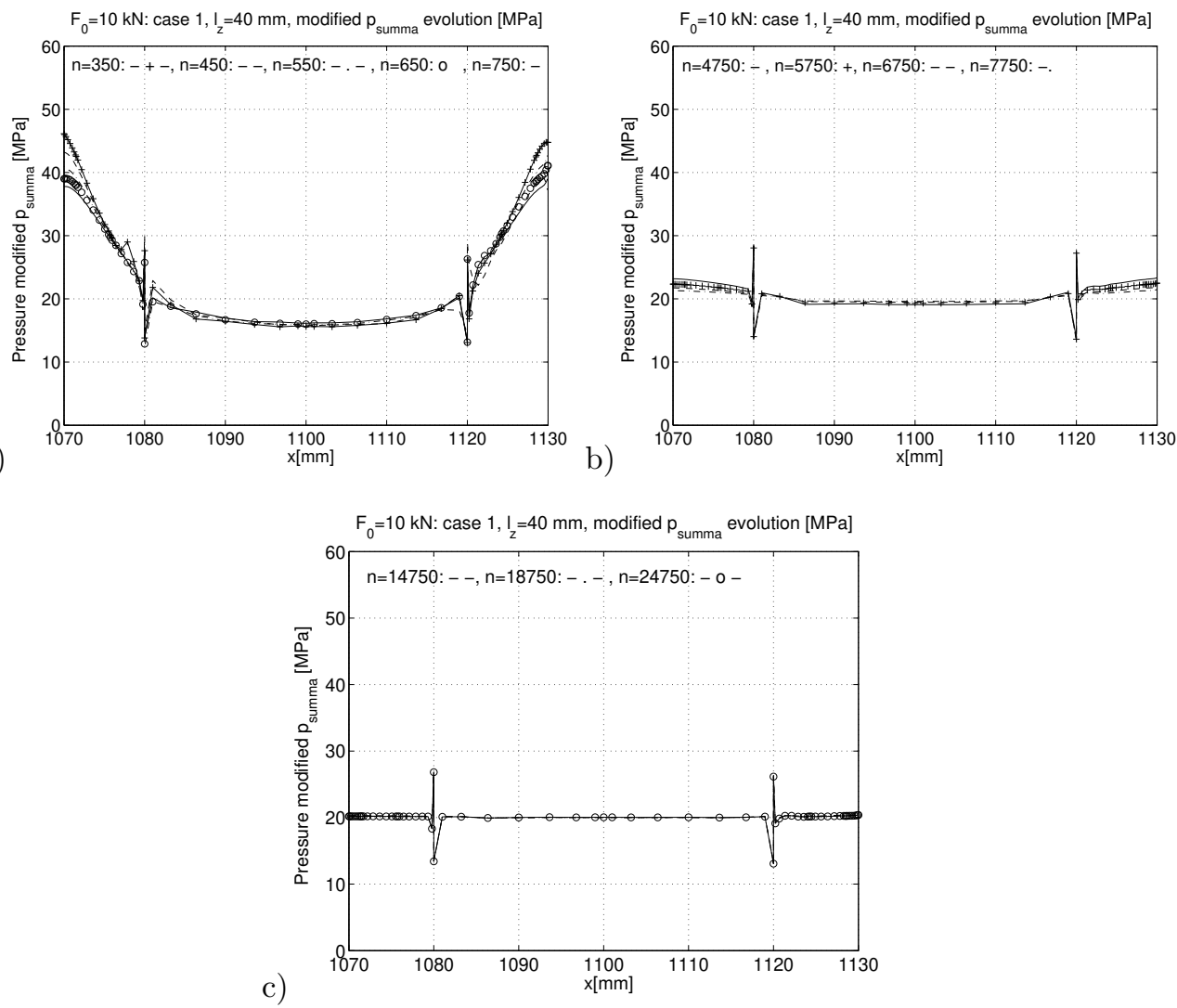

Figure 25. Modified sum of contact pressures in one cycle

4.2. Non-symmetric load (Load case 2). Let us now analyze the case of eccentric load when the resultant vertical force is $F=10 \mathrm{kN}$. The pressure $p^{\sim}=20 \mathrm{MPa}$ is applied in the interval $10 \leq \widetilde{z} \leq 60$. The resultant position coordinate is $\widetilde{z}_{F}=35 \mathrm{~mm}$.

Solving 4.18 we find $p_{m}^{C}=3.572 \mathrm{MPa}, p_{m}^{L}=0.2143 \mathrm{MPa} / \mathrm{mm}$.

The numerical analysis results are shown in Figures 26 29. They demonstrate a good approximation of the theoretical values of the modified summed contact pressure. The maximum of difference is smaller than $5 \%$ (see Figure 29. 
a)
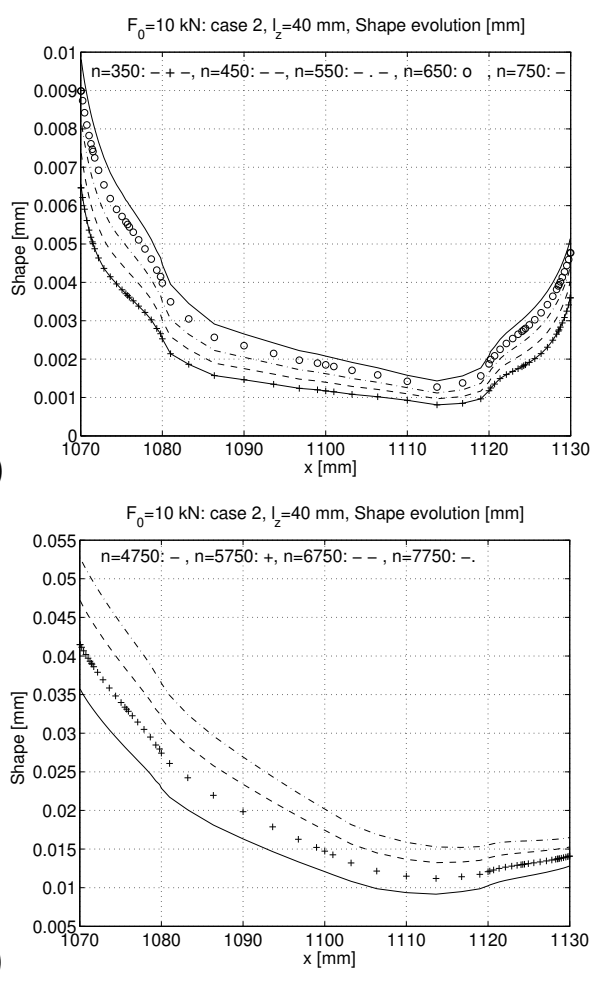

b)

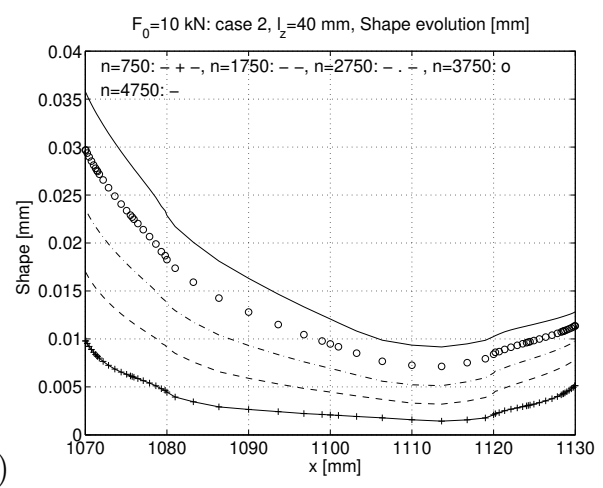

d)

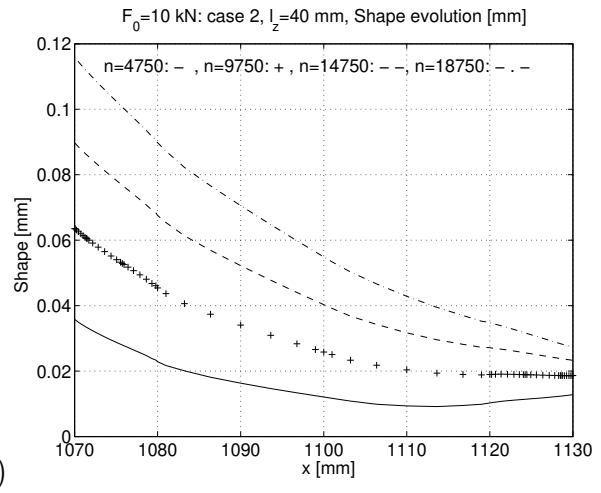

Figure 26. Shape evolution in the wear process

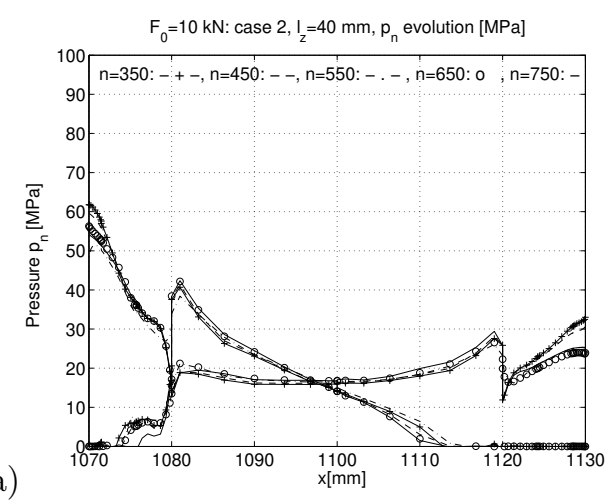

b)

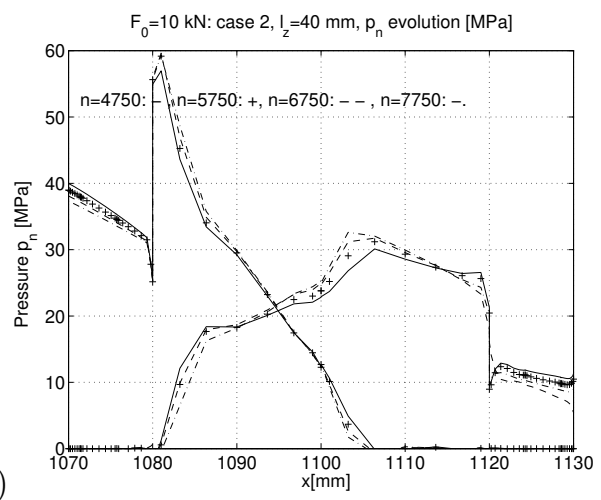

Figure 27. Contact pressure at different time steps 
a)
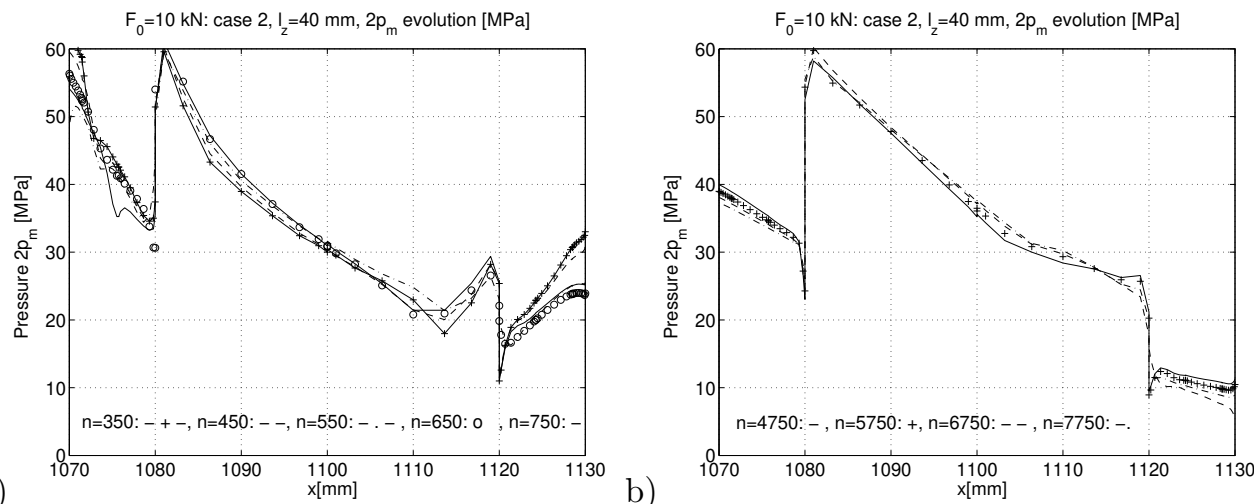

b)

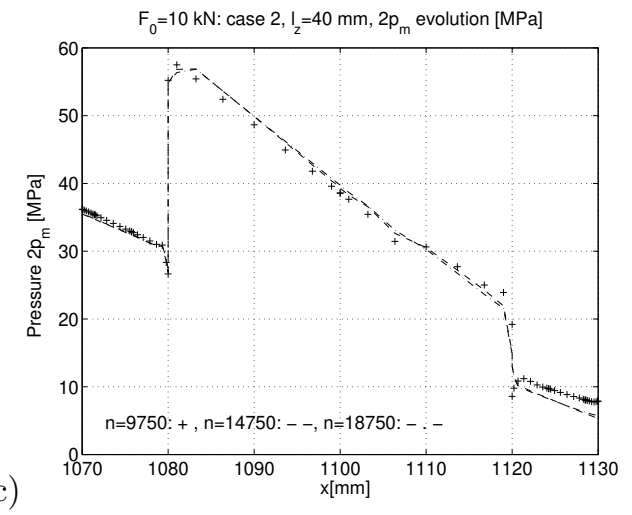

Figure 28. Summa of contact pressures in one cycle

At the interface of different material the stress state exhibits a singularity, which is manifested by a discontinuity of the contact stresses (see Figures 27 29).

\section{STEADy STATE CONDITIONS FOR PERIOdIC LOADING}

Consider now the steady state wear problem for the case of a punch sliding monotonically on a flat substrate and subjected to periodic normal loading. The steady state conditions at each point of the contact zone are now expressed as follows:

$$
u_{\tau}(t)=u_{\tau}\left(t+T_{*}\right), \quad \boldsymbol{\sigma}(t)=\boldsymbol{\sigma}\left(t+T_{*}\right), \quad \varepsilon(t)=\varepsilon\left(t+T_{*}\right),
$$

where $T_{*}$ is the period of loading, $\boldsymbol{\sigma}$ and $\varepsilon$ are the stress and the strain fields. The related cyclic wear increment accumulated during one cycle is assumed to be compatible with the rigid body wear motion of punch, thus

$$
w_{n}\left(x, t+T_{*}\right)-w_{n}(x, t)=\Delta w_{n}\left(x, T_{*}\right)=\left(\Delta \boldsymbol{\lambda}_{F}+\Delta \boldsymbol{\lambda}_{M} \times \Delta \boldsymbol{r}\right) \cdot \boldsymbol{n}_{c},
$$

where $\Delta \boldsymbol{\lambda}_{F}$ and $\Delta \boldsymbol{\lambda}_{M}$ are the translational and rotational cyclic wear increments. 
a)
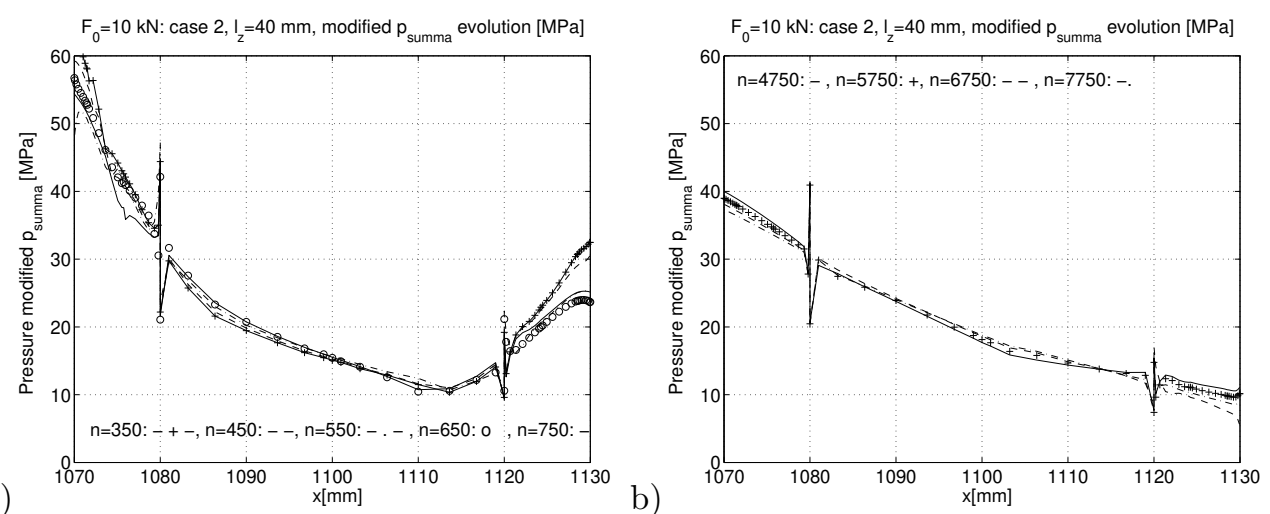

b)

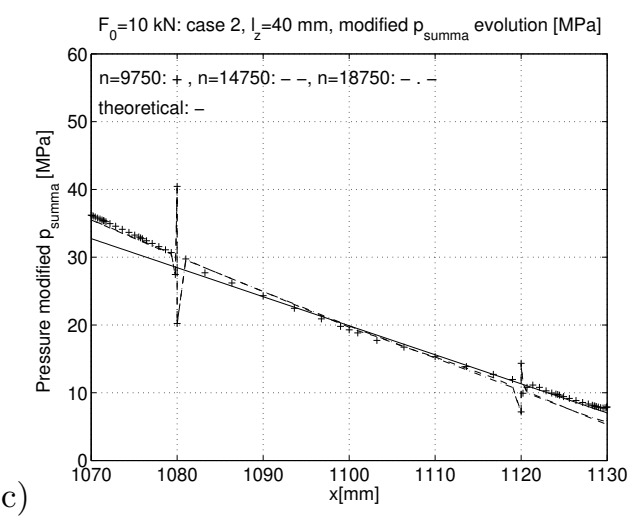

Figure 29. Modified sum of contact pressures in one cycle

For the simplification of calculations of the relative velocity between the bodies it is assumed that

$$
\dot{\boldsymbol{u}}_{\tau}=\dot{\boldsymbol{u}}_{R, \tau}^{(2)}-\dot{\boldsymbol{u}}_{R, \tau}^{(1)}=\dot{\boldsymbol{u}}_{R, \tau}=-\left\|\dot{\boldsymbol{u}}_{\tau}\right\| \boldsymbol{e}_{\tau 1}=-v_{r} \boldsymbol{e}_{\tau 1}=-v_{r} \boldsymbol{e}_{x}
$$

since the elastic and wear relative velocities are very small as compared to specified rigid body velocities $\dot{\boldsymbol{u}}_{R, \tau}^{(1)}$ and $\dot{\boldsymbol{u}}_{R, \tau}^{(2)}$ generating the sliding regime

$$
\left\|\dot{\boldsymbol{u}}_{e, \tau}\right\|=\left\|\dot{\boldsymbol{u}}_{e, \tau}^{(2)}-\dot{\boldsymbol{u}}_{e, \tau}^{(1)}+\dot{\boldsymbol{w}}_{2, \tau}-\dot{\boldsymbol{w}}_{1, \tau}\right\| \ll\left\|\dot{\boldsymbol{u}}_{R, \tau}\right\|=\left\|\dot{\boldsymbol{u}}_{R, \tau}^{(2)}-\dot{\boldsymbol{u}}_{R, \tau}^{(1)}\right\|
$$

The substrate is assumed to slide monotonically in the leftward direction. The analysis presented pertains to the case when wear of punch or combined wear of punch and substrate occurs. Then the cyclic wear increment at the contact is controlled by the rigid body motion of one of the contacting bodies. The contact shape evolution during consecutive cycles assures stress and strain periodicity. 


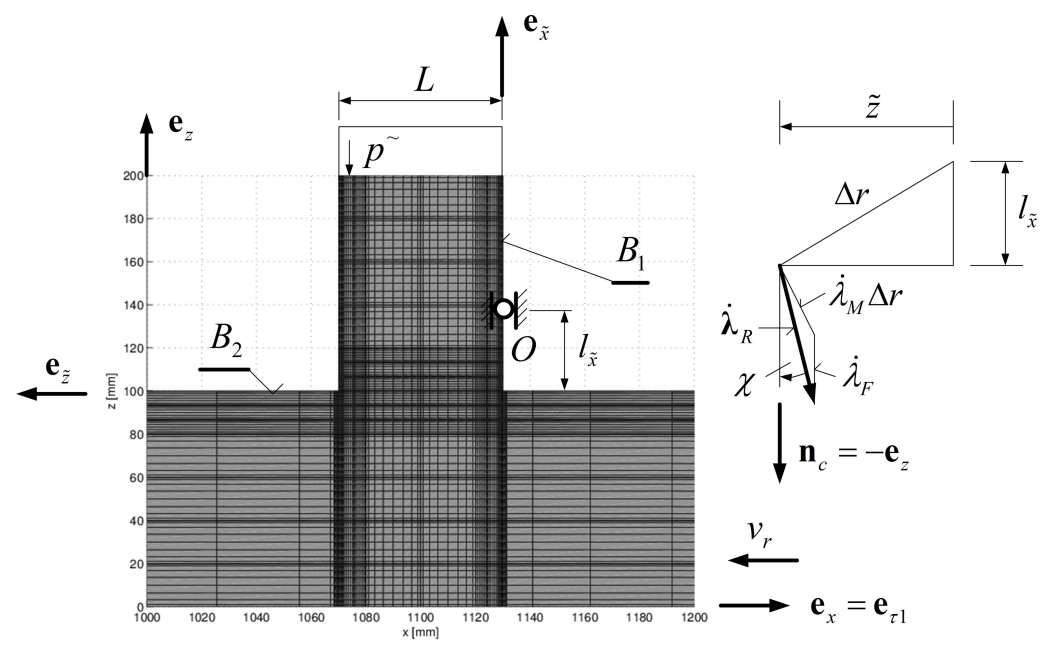

Figure 30. Mesh of punch constrained at $O$ and of central part of the strip. Rigid body wear velocity $\boldsymbol{\lambda}_{R}$. Strip is moving in leftward direction.

To simplify the analysis, only the case of punch wear is considered and the wear parameters are $\widetilde{\beta}_{1} \neq 0, \widetilde{\beta}_{2}=0, a=b=1$. In paper 6 it was supposed that wear parameters are uniform. In the following it is assumed that wear parameters are non-uniform.

Consider the periodic loading applied to punch $B_{1}$ in the direction $-\boldsymbol{e}_{z}$. (see Figure 30. The resultant vertical load is now expressed as

$$
\boldsymbol{F}=-F \boldsymbol{e}_{z}=F=F_{0}+F_{A}|\sin \omega \tau| \geqslant 0,
$$

where $F_{0}$ is the constant load, $F_{A}$ and $\omega$ are the load amplitude and frequency. In view of the constraint at $O$, the load generates the resulting moment $M_{0}^{y}$ with respect to the $y$ axis. The period of loading is $T_{*}=2 \pi / \omega$.

The loading on the upper punch boundary by uniform pressure $p^{\sim}$ is applied with the resulting load $F$. Referring to Figure 30, it is assumed that during the wear process the punch is allowed to translate in the normal contact direction and rotate around the constraining pin $O$.

The average load value in one cycle is

$$
\bar{F}=\frac{1}{T_{*}} \int_{0}^{T_{*}} F \mathrm{~d} \tau=F_{0}+\frac{2}{\pi} F_{A} .
$$

The integrated wear velocity at the position $x$ of the contact zone in one loading period is

$$
\Delta \boldsymbol{\lambda}_{F, M}(x)=\int_{0}^{T_{*}} \dot{\lambda}_{F, M}(x, \tau) \mathrm{d} \tau
$$


and the average wear velocity field is expressed as follows

$$
\overline{\dot{\lambda}}_{F, M}(x)=\frac{\Delta \boldsymbol{\lambda}_{F, M}}{T_{*}}=\frac{1}{T_{*}} \int_{0}^{T_{*}} \dot{\boldsymbol{\lambda}}_{F, M}(x, \tau) \mathrm{d} \tau .
$$

The cyclic wear increment for the punch allowed to translate in the normal contact direction is

$$
\Delta w_{1, n}(x)=\int_{0}^{T_{*}} \widetilde{\beta}_{1}(x) p_{n}(x, \tau) v_{r} \mathrm{~d} \tau=\widetilde{\beta}_{1}(x) v_{r} \int_{0}^{T_{*}} p_{n}(x, \tau) \mathrm{d} \tau
$$

and the average value in one period is

$$
\Delta \bar{w}_{1, n}(x)=\frac{\Delta w_{1, n}(x)}{T_{*}}=\widetilde{\beta}_{1}(x) v_{r} \frac{\int_{0}^{T_{*}} p_{n}(x, \tau) \mathrm{d} \tau}{T_{*}} .
$$

The wear volume in one period equals

$$
\begin{aligned}
\Delta W=\int_{S_{c}} \Delta w_{1, n}(x) \mathrm{d} S=\int_{S_{c}} \int_{0}^{T_{*}} \widetilde{\beta}_{1}(x) p_{n}(x, \tau) v_{r} \mathrm{~d} \tau \mathrm{d} S= \\
\qquad v_{r} \int_{0}^{T_{*}} \int_{S_{c}} \widetilde{\beta}_{1}(x) p_{n}(x, \tau) \mathrm{d} S \mathrm{~d} \tau=v_{r} \int_{0}^{T_{*}} F_{\beta}(\tau) \mathrm{d} \tau
\end{aligned}
$$

since from the equilibrium equation it follows that $\int_{S_{c}} \widetilde{\beta}_{1}(x) p_{n}(x, \tau) \mathrm{d} S=F_{\beta}(\tau)$ and the average wear volume increment is

$$
\Delta \bar{W}=\frac{\Delta W}{T_{*}}=\frac{v_{r}}{T_{*}} \int_{0}^{T_{*}} F_{\beta}(\tau) \mathrm{d} \tau=v_{r} \bar{F}_{\beta} .
$$

It is seen that $\Delta \bar{W}$ is explicitly related to the average modified load value $\bar{F}_{\beta}$. The contact traction vector in our case is expressed as $\boldsymbol{t}^{c}=\boldsymbol{t}_{1}^{c}=-\boldsymbol{t}_{2}^{c}=p_{n} \boldsymbol{e}_{z}-\mu p_{n} \boldsymbol{e}_{x}$, the wear velocity vectors are

$$
\dot{\boldsymbol{w}}_{R}=\dot{\boldsymbol{w}}_{2}-\dot{\boldsymbol{w}}_{1}, \dot{\boldsymbol{w}}_{i}=(-1)^{i}\left[-\dot{w}_{i, n} \boldsymbol{e}_{z}+\dot{w}_{i, \tau} \boldsymbol{e}_{x}\right], \quad i=1,2,
$$

since the relative velocity is

$$
\dot{\boldsymbol{u}}_{\tau}=\dot{\boldsymbol{u}}_{x}=\dot{\boldsymbol{u}}_{2, x}-\dot{\boldsymbol{u}}_{1, x}=-v_{r} \boldsymbol{e}_{\tau 1}=-v_{r} \boldsymbol{e}_{x}
$$

and $\dot{w}_{i, \tau}=\dot{w}_{i, n} \tan \chi, 6$. The punch width is equal to $L$. The thickness of two bodies is $t_{\mathrm{th}}$, so the contact zone area equals $S_{c}=t_{\mathrm{th}} L$. The position of the pin $O$ in the vertical direction is denoted by $l_{\widetilde{x}}$ (see Figure 30 . Let $\widetilde{z}=1130-x$ denote the typical contact point position relative to the right perimeter of contact zone. 
Let us define the wear dissipation in one cycle of sliding motion

$$
E_{w}=\frac{1}{2} \int_{0}^{T_{*}}\left(\int_{S_{c}} \boldsymbol{t}_{1}^{c} \cdot \dot{\boldsymbol{w}}_{1} \mathrm{~d} S\right) \mathrm{d} \tau
$$

and the equilibrium equations for the punch

$$
\begin{aligned}
F-\int_{S_{c}} p_{n} \mathrm{~d} S & =0, \\
M-\int_{S_{c}} p_{n}\left[\widetilde{z}+\mu l_{\widetilde{x}}\right] \mathrm{d} S & =0
\end{aligned}
$$

where $\widetilde{z}_{F}$ is the position coordinate of the resultant load $F$.

The steady state conditions will be determined by minimizing the average wear dissipation in one cycle subject to punch equilibrium equations (5.14). The Lagrangian averaged functional is now expressed as follows

$$
\begin{aligned}
\bar{L}_{w}= & \frac{E_{w}}{T_{*}}-\frac{1}{T_{*}} \int_{0}^{T_{*}} \dot{\lambda}_{F}\left(\int_{S_{c}} p_{n} \mathrm{~d} S-F\right) \mathrm{d} \tau- \\
& \frac{1}{T_{*}} \int_{0}^{T_{*}} \dot{\lambda}_{M}\left\{\int_{S_{c}} p_{n}\left[\widetilde{z}+\mu l_{\widetilde{x}}\right] \mathrm{d} S-M\right\} \mathrm{d} \tau
\end{aligned}
$$

where in view of $(5.13)$, there is

$$
E_{w}=\frac{1}{2} \int_{0}^{T_{*}} \int_{S_{c}} \widetilde{\beta}_{1} v_{r}\left(p_{n}\right)^{2}\{1+\mu \tan \chi\} \mathrm{d} S \mathrm{~d} \tau
$$

and $\chi$ is the orientation angle of the wear velocity vector relative to the normal contact vector, thus $\tan \chi=\frac{\dot{\lambda}_{M} l_{\widetilde{x}}}{\dot{\lambda}_{F}+\dot{\lambda}_{M} \widetilde{z}}$, because

$$
\dot{\boldsymbol{w}}_{R}=-\dot{\boldsymbol{w}}_{1}, \dot{\boldsymbol{w}}_{1}=-\left[\dot{\lambda}_{F}+\dot{\lambda}_{M} \widetilde{z}\right] \boldsymbol{e}_{z}-\dot{\lambda}_{M} l_{\widetilde{x}} \boldsymbol{e}_{x}, \quad \boldsymbol{e}_{R}=\frac{\dot{\boldsymbol{w}}_{R}}{\left\|\dot{\boldsymbol{w}}_{R}\right\|}
$$

(see Figure 30).

The Lagrangian multipliers represent the rigid body wear velocity components. Considering the variation of (5.15) and (5.16), the following expression is obtained:

$$
\begin{aligned}
& \delta \bar{L}_{w}=\frac{1}{T_{*}} \int_{0}^{T_{*}} \int_{S_{c}}\left\{\widetilde{\beta}_{1} v_{r} p_{n}\{1+\mu \tan \chi\}-\dot{\lambda}_{F}-\dot{\lambda}_{M}\left(\widetilde{z}+\mu l_{\widetilde{x}}\right)\right\} \delta p_{n} \mathrm{~d} S \mathrm{~d} \tau- \\
& -\frac{1}{T_{*}} \int_{0}^{T_{*}} \delta \dot{\lambda}_{F}\left(\int_{S_{c}} p_{n} \mathrm{~d} S-F\right) \mathrm{d} \tau-\frac{1}{T_{*}} \int_{0}^{T_{*}} \delta \dot{\lambda}_{M}\left(\int_{S_{c}}\left(\widetilde{z}+\mu l_{\widetilde{x}}\right) p_{n} \mathrm{~d} S-M\right) \mathrm{d} \tau=0 .
\end{aligned}
$$


Since the variations of contact pressure and of the rigid body wear velocity are arbitrary, the relative velocity $v_{r}$ is independent of time and can be written

$$
\begin{aligned}
\delta \bar{L}_{w}=\int_{S_{c}}\left\{\widetilde{\beta}_{1} v_{r} \bar{p}_{n}\{1+\mu \tan \chi\}-\overline{\dot{\lambda}}_{F}-\overline{\dot{\lambda}}_{M}\left(\widetilde{z}+\mu l_{\widetilde{x}}\right)\right\} \delta p_{n} \mathrm{~d} S- \\
\quad-\delta \dot{\lambda}_{F}\left(\int_{S_{c}} \bar{p}_{n} \mathrm{~d} S-\bar{F}\right)-\delta \dot{\lambda}_{M}\left(\int_{S_{c}}\left(\widetilde{z}+\mu l_{\widetilde{x}}\right) \bar{p}_{n} \mathrm{~d} S-\bar{M}\right)=0 .
\end{aligned}
$$

First, we determine the contact average pressure related to wear velocity components

$$
\bar{p}_{n}=\frac{\overline{\dot{\lambda}}_{F}+\overline{\dot{\lambda}}_{M} \widetilde{z}}{\widetilde{\beta}_{1} v_{r}}
$$

and then from the equilibrium equations for average states we have

$$
\int_{S_{c}} \bar{p}_{n} \mathrm{~d} S-\bar{F}=0, \quad \int_{S_{c}}\left(\widetilde{z}+\mu l_{\widetilde{x}}\right) \bar{p}_{n} \mathrm{~d} S-\bar{M}=0,
$$

where $\bar{F}$ is calculated from 5.6 . From 5.19 it follows that modified contact pressure $\widetilde{\beta}_{1}(\widetilde{z}) \bar{p}_{n}(\widetilde{z})$ is a linear function of the $\widetilde{z}$, but the pressure at different positions in the contact zone, where the wear parameter is constant: $\widetilde{\beta}_{1}(\widetilde{z})=\widetilde{\beta}_{s}$ or $\widetilde{\beta}_{1}(\widetilde{z})=\widetilde{\beta}_{m}$ is also linear, with differing inclination (see Figure 31.

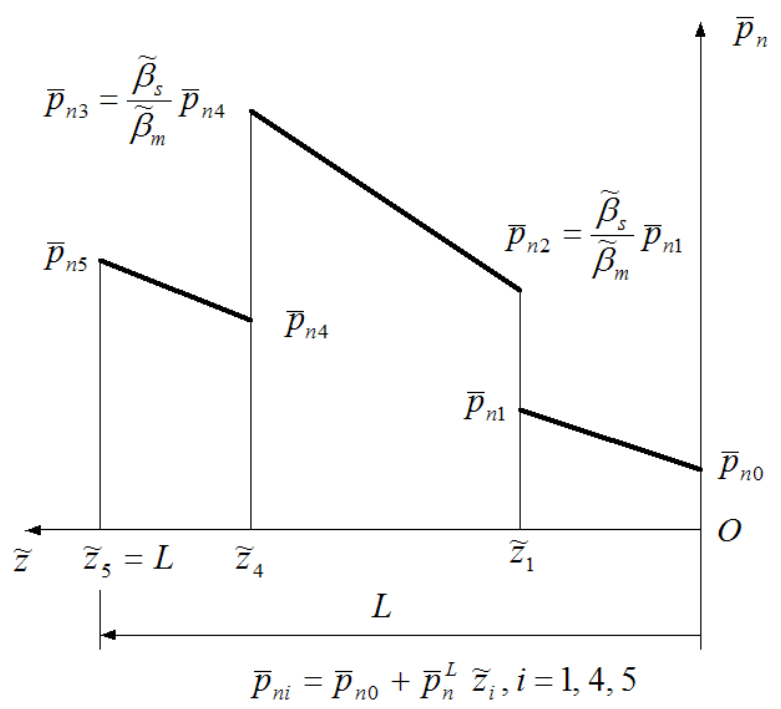

Figure 31. Average contact pressure distribution in the contact zone

When the wear parameters vary along the contact domain, then referring to Figure 31. the following system of algebraic equations for average rigid wear velocities is 
used:

$$
\left[\begin{array}{ll}
B_{11} & B_{12} \\
B_{21} & B_{22}
\end{array}\right]\left[\begin{array}{l}
\overline{\dot{\lambda}}_{F} \\
\dot{\dot{\lambda}}_{M}
\end{array}\right]=\frac{1}{t_{\mathrm{th}}}\left[\frac{\bar{F}}{\bar{M}}\right] \widetilde{\beta}_{s} v_{r}
$$

where

$$
\begin{gathered}
B_{11}=\widetilde{z}_{1}+\frac{\widetilde{\beta}_{s}}{\widetilde{\beta}_{m}}\left(\widetilde{z}_{4}-\widetilde{z}_{1}\right)+\widetilde{z}_{5}-\widetilde{z}_{4}, \quad B_{12}=\frac{1}{2}\left[\widetilde{z}_{1}^{2}+\frac{\widetilde{\beta}_{s}}{\widetilde{\beta}_{m}}\left(\widetilde{z}_{4}^{2}-\widetilde{z}_{1}^{2}\right)+\widetilde{z}_{5}^{2}-\widetilde{z}_{4}^{2}\right] \\
B_{21}=B_{12}+\mu l_{\widetilde{x}} B_{11}, \quad B_{22}=\frac{1}{3}\left[\widetilde{z}_{1}^{3}+\frac{\widetilde{\beta}_{s}}{\widetilde{\beta}_{m}}\left(\widetilde{z}_{4}^{3}-\widetilde{z}_{1}^{3}\right)+\widetilde{z}_{5}^{3}-\widetilde{z}_{4}^{3}\right]+\mu l_{\widetilde{x}} B_{12} .
\end{gathered}
$$

Solving (5.21), the contact pressures are expressed by the relations

$$
\begin{aligned}
& \bar{p}_{n}=\frac{\overline{\dot{\lambda}}_{F}+\overline{\dot{\lambda}}_{M} \widetilde{z}}{\widetilde{\beta}_{s} v_{r}} \quad \text { if } \quad 0 \leq \widetilde{z} \leq \widetilde{z}_{1}, \quad \widetilde{z}_{4} \leq \widetilde{z} \leq \widetilde{z}_{5} ; \\
& \bar{p}_{n}=\frac{\overline{\dot{\lambda}}_{F}+\overline{\dot{\lambda}}_{M} \widetilde{z}}{\widetilde{\beta}_{m} v_{r}} \quad \text { if } \quad \widetilde{z}_{1} \leq \widetilde{z} \leq \widetilde{z}_{4} .
\end{aligned}
$$

The average rigid body wear velocities for the case of uniform wear parameter $\widetilde{\beta}_{1}(\widetilde{z})=$ const are expressed in the form

$$
\overline{\dot{\lambda}}_{F}=\widetilde{\beta}_{1} v_{r} \frac{\bar{F}}{S_{c}}\left[1-\frac{6}{L}\left(z_{F}-\frac{L}{2}-\mu l_{\widetilde{x}}\right)\right], \overline{\dot{\lambda}}_{M}=\widetilde{\beta}_{1} v_{r} \frac{\bar{F}}{S_{c}} \frac{12}{L^{2}}\left(z_{F}-\frac{L}{2}-\mu l_{\widetilde{x}}\right)
$$

and the average contact pressure distribution is

$$
\bar{p}_{n}=\frac{\bar{F}}{S_{c}}\left\{1+\left(-\frac{6}{L}+\frac{12}{L^{2}} \widetilde{z}\right)\left(z_{F}-\frac{L}{2}-\mu l_{\widetilde{x}}\right)\right\} .
$$

The analysis presented referred to the specific problem of a plane punch sliding on a flat substrate. However, the method can be applied to any case of the relative sliding of two bodies for periodically varying normal load. We can therefore make a general statement.

Theorem. For the cases of periodic normal loading and monotonic relative sliding between two bodies, the average contact wear form and pressure distribution can be specified in terms of the average load from the minimization of the wear dissipation power in one period.

Remark 1: If the punch is not allowed for rigid body rotation $\left(\dot{\lambda}_{M}=0, l_{\widetilde{x}}=0\right.$, $\widetilde{z}_{F}=L / 2$ ), and wear parameters are uniform, then

$$
\bar{p}_{n}=\bar{F} / S_{c}, S_{c}=t_{\mathrm{th}} \widetilde{z}_{5}=t_{\mathrm{th}} L .
$$

If wear parameters are not uniform then $\overline{\dot{\lambda}}_{F}=\frac{\bar{F}}{t_{\mathrm{th}} B_{11}} \widetilde{\beta}_{s} v_{r}$. The contact pressure then is

$\bar{p}_{n}=\frac{\bar{F}}{t_{\mathrm{th}} B_{11}} \quad$ if $\quad 0 \leq \widetilde{z} \leq \widetilde{z}_{1}, \quad \widetilde{z}_{4} \leq \widetilde{z} \leq \widetilde{z}_{5} \quad$ and $\quad \bar{p}_{n}=\frac{\bar{F}}{t_{\mathrm{th}} B_{11}} \frac{\widetilde{\beta}_{s}}{\widetilde{\beta}_{m}} \quad$ if $\quad \widetilde{z}_{1} \leq \widetilde{z} \leq \widetilde{z}_{4}$. 
Remark 2: When the relative sliding velocity depends on time (for instance varies periodically), then in variational equations (5.17) and 5.18 the term $\frac{1}{T_{*}} \int_{0}^{T_{*}} v_{r} p_{n} \mathrm{~d} \tau=$ $\overline{v_{r} p_{n}}=\bar{v}_{r} \bar{p}_{n}+\frac{1}{T_{*}} \int_{0}^{T_{*}} \widetilde{v}_{r} \widetilde{p}_{n} \mathrm{~d} \tau$, where $\widetilde{v}_{r}$ and $\widetilde{p}_{n}$ are the fluctuation values, so the result for the average contact pressure $(5.22)$ will only be approximate.

\section{Concluding Remarks}

The present paper also demonstrated that the minimum of the wear dissipation power at $q=1$ gives the pressure distribution of the steady wear state at arbitrary wear parameters. When the heat generation on the contact surface is accounted for, the contact pressure is the same as in the case when the heat generation is neglected, but the steady wear contact shape is totally different.

In the case of periodic sliding, the modified sum of contact pressures is determined and used to specify the accumulated wear, with no need for time integration of the wear rule.

Acknowledgement. The present research was partially supported by the Hungarian Academy of Sciences, by grant NKFIH 115701.

\section{REFERENCES}

1. I. Páczelt and Z. Mróz. "On the analysis of steady sliding wear process." Tribology International Journal, 42, (2009), pp. 275-283. DOI: 10.1016/j.triboint. 2008.06.007.

2. I. Páczelt and Z. Mróz. "Numerical analysis of steady thermo-elastic wear regimes induced by translating and rotating punches." Computers and Structures, 89, (2011), pp. 2495-2521. DOI: 10.1016/j .compstruc.2011.06.001.

3. I. Páczelt and Z. Mróz. "Optimal shapes of contact interfaces due to sliding wear in the steady relative motion." International Journal of Solids and Structures, 44, (2007), pp. 895-925. DOI: 10.1016/j.ijsolstr.2006.05.027.

4. Z. Mróz and I. Páczelt. "Analysis of Thermo-Elastic Wear Problems." Journal of Thermal Stresses, 34, (2011), pp. 569-606. DOI: 10.1080/01495739.2011. 564026 .

5. I. Páczelt, Z. Mróz, and A. Baksa. "Analysis of steady wear processes for periodic sliding." Journal of Computational and Applied Mechanics, 10, (2015), pp. 231268. DOI: $10.32973 /$ jcam.2015.014.

6. I. Páczelt and Z. Mróz. "Analysis of wear processes for periodic loading." Key Engineering Materials, 681, (2015), pp. 117-141. DOI:10.4028/www.scientific. net/KEM.681.117.

7. Z. Mróz, S. Kucharski, and I. Páczelt. "Anisotropic friction and wear rules with account for contact state evolution." Wear, 396-397, (2018), pp. 1-11. DOI: $10.1016 / \mathrm{j}$. wear.2017.11.004

8. I. G. Goryacheva. Contact Mechanics in Tribology. Dordrecht, Kluwer Academic Publishers, 2010. DOI: doi:10.1007/978-94-015-9048-8. 
9. B. Szabó and I. Babuska. Introduction to Finite Element Analysis : Formulation, Verification and Validation. New York, Wiley-Interscience, 2011.

10. I. Páczelt and A. Baksa. "Examination of contact optimization and wearing problems." Journal of Computational and Applied Mechanics, 3, (2002), pp. 6184. URL: http://www.mech.uni-miskolc.hu/jcam.

11. P. Wriggers. Computational Contact Mechanics. Springer, 2006. DOI: 10.1007/ 978-94-015-9048-8.

12. I. Páczelt. "Iterative methods for solution of contact optimization problems." Archives of Mechanics, 52, (2000), pp. 685-711. URL: http://am.ippt.pan . $\mathrm{pl/am/article/view/v52p685.}$

13. U. Peigney. "Simulating wear under cyclic loading by a minimization approach." International Journal of Solids and Structures, 41, (2004), pp. 6783-6799. DOI: 10.1016/j.ijsolstr.2004.05.022.

14. N. H. Kim, D. Won, D. Burris, B. Holtkamp, G. C. Gessel, P. Swanson, and W. G. Sawyer. "Finite element analysis and experiments of metal/metal wear in oscillatory contacts." Wear, 258, (2005), pp. 1787-1793. DOI: 10.1016/j .wear. 2004.12.014.

15. I. G. Goryacheva, P. T. Rajeev, and T. N. Farris. "Wear in partial slip contact." Journal of Tribology ASME, 123, (2001), pp. 848-856. DOI: 10.1115/1.1338476.

16. I. Páczelt and Z. Mróz. "Analysis of thermo-mechanical wear problems for reciprocal punch sliding." Advances in Engineering Software, 80, (2015), pp. 139 155. DOI: $10.1016 / \mathrm{j}$.advengsoft.2014.09.012 